\title{
PHASE RETRIEVAL IN FRAME THEORY
}

A Dissertation presented to

the Faculty of the Graduate School

at the University of Missouri

In Partial Fulfillment

of the Requirements for the Degree

Doctor of Philosophy

by
DORSA GHOREISHI

Dr. Peter G. Casazza, Dissertation Supervisor

MAY 2020 
The undersigned, appointed by the Dean of the Graduate School, have examined the thesis entitled:

\section{PHASE RETRIEVAL IN}

\section{FRAME THEORY}

presented by Dorsa Ghoreishi, a candidate for the degree of Doctor of Philosophy and hereby certify that, in their opinion, it is worthy of acceptance.

Professor Peter G. Casazza

Professor Stephen Montgomery-Smith

Professor Nakhle Asmar

Professor David Retzloff 


\section{ACKNOWLEDGMENTS}

I would first like to thank my advisor Peter Casazza for his vision and guidance during my program. This was not possible without him. He and Janet are among the most generous and kind people I have had a pleasure of knowing.

I would also like to thank my committee members Dr. Nakhle Asmar, Dr. Stephen Montgomery-Smith, and Dr. David Retzloff for taking their time to be on my committee. I would also like to thank Dr. Petros Valettas for his insight and guidance.

I am grateful to my parents for their endless support and for always encouraging me to pursue my goals and dreams. Also, my amazing sister who was always my guidance and motivation to continue this path.

I also want to thank my incredible friends who have helped to make graduate school an amazing experience by their encouragement and cheers. 


\section{TABLE OF CONTENTS}

ACKNOWLEDGMENTS ................ ii

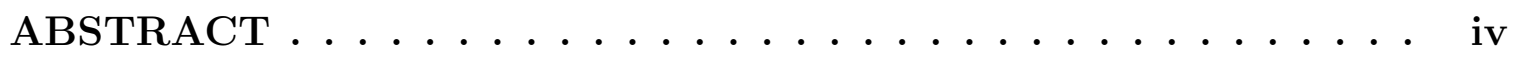

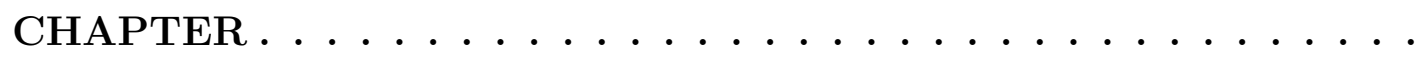

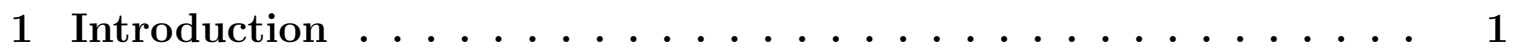

1.0 .1 Frames. . . . . . . . . . . . . . . . 2

2 Phase Retrieval and Norm Retrieval . . . . . . . . . . . 9

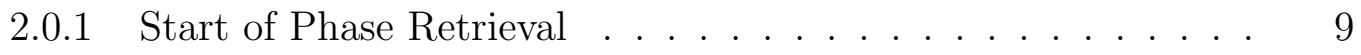

2.0.2 Main Problems and Definitions _. . . . . . . . 10

2.0 .3 Subspaces and Projections . . . . . . . . . . . . . 14

2.0 .4 Norm Retrieval . . . . . . . . . . . . . . . . . . 17

2.0.5 Phase Retrieval and Norm Retrieval . . . . . . . . . . . 26

2.0.6 Classification of Norm Retrieval . . . . . . . . . . . . 33

3 Weak Phase Retrieval $\ldots \ldots \ldots \ldots$

3.1 Defining weak phase retrieval $\ldots \ldots \ldots \ldots \ldots$

$3.1 .1 \quad$ Real Case . . . . . . . . . . . . . . . . . . . . 44

3.1 .2 Complex Case . . . . . . . . . . . . . . . . . . 51

3.2 Weak Phaseless Reconstruction . . . . . . . . . . . . . . 52

3.3 Illustrative Examples . . . . . . . . . . . . . . . . . . 58

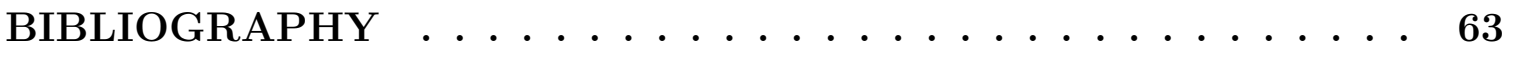

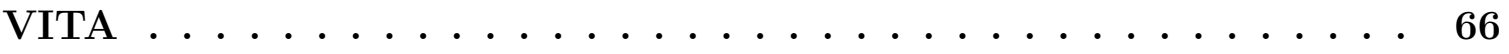




\title{
PHASE RETRIEVAL IN FRAME THEORY
}

\section{Dorsa Ghoreishi}

Dr. Peter G. Casazza, Dissertation Suppervisor

\begin{abstract}
This dissertation is the study of phase retrieval in frame theory. The first part is concerned with the analysis of phase retrieval and the complete classification of norm retrieval. Norm retrieval is essential to transfer the properties of phase retrieval to the complement space. The first section includes the results regarding projections and also the characterization of phase retrieval and norm retrieval for subspaces. The second part is the study of weak phase retrieval which was motivated by the idea of reducing the number of vectors satisfying the properties close to phase retrieval. The last section provides the correlation between weak phase retrieval and phase retrieval properties along with the examples illustrating the relationship between weak phase retrieval and the related concepts.
\end{abstract}




\section{Chapter 1}

\section{Introduction}

Frames for Hilbert space were formally introduced by Duffin and Schaeffer in 1952 while studying deep questions in non-harmonic Fourier series. Frames have been used in noise and erasure reduction, compressed sensing, sampling theory, data quantization, quantum measurements, coding, image processing, wireless communications, time-frequency analysis, speech recognition, bio-imaging, and much more. Nowadays, frames have broad application to problems in pure mathematics, applied mathematics, engineering and more. Frame theory has proven to be a powerful area of research with applications. One of the problems we can ask in frame theory is how to classify and reconstruct frames to be able to use them in more applied problems. This can often be very difficult if not impossible in practice.

This dissertation is dedicated to the study of phase retrieval properties for the applications in different problems such as engineering problems. We will provide the classifications for phase retrieval, norm retrieval and weak phase retrieval by developing and generalizing the known results and distributing them to completely study the norm retrieval properties over subspaces and in the complement space as well as defining the weak phase retrieval property. 


\subsubsection{Frames}

In this section, we introduce some of the basic definitions and results from frame theory. Throughout this dissertation, $\mathbb{H}^{m}$ denotes an $m$ dimensional real or complex Hilbert space and we will write $\mathbb{R}^{m}$ or $\mathbb{C}^{m}$ when it is necessary to differentiate between the two. We start with the definition of a frame in $\mathbb{H}^{m}$.

Definition 1.0.1. A family of vectors $\Phi=\left\{\phi_{i}\right\}_{i=1}^{n}$ in $\mathbb{H}^{m}$ is a frame if there are constants $0<A \leq B<\infty$ so that for all $x \in \mathbb{H}^{m}$

$$
A\|x\|^{2} \leq \sum_{i=1}^{n}\left|\left\langle x, \phi_{i}\right\rangle\right|^{2} \leq B\|x\|^{2},
$$

we have:

1. $A$ and $B$ are the lower and upper frame bounds of the frame

2. The frame is called an A-tight frame if $A=B$

3. The frame is called a Parseval frame if $A=B=1$

4. If we only assume $0<B<\infty$ then it is called a $\mathrm{B}$-Bessel sequence

5. $\Phi$ is called an equal norm frame if $\left\|\phi_{i}\right\|=\left\|\phi_{j}\right\|$ for all $i, j$ and is called a unit norm frame if $\left\|\phi_{i}\right\|=1$ for all $i \in[n]$

In finite dimensions, the definition of a frame is equivalent to a spanning set. However, in infinite dimensions there are examples of spanning sets which do not satisfy the frame inequality. It is important to note that there are no restrictions on the frame vectors. For example, if $\left\{e_{i}\right\}_{i=1}^{m}$ is an orthonormal basis for $\mathbb{H}^{m}$, then

$$
\left\{e_{1}, 0, e_{2}, 0, \ldots, 0, e_{m}\right\}
$$


and

$$
\left\{e_{1}, \frac{e_{2}}{\sqrt{2}}, \frac{e_{2}}{\sqrt{2}}, \frac{e_{3}}{\sqrt{3}}, \frac{e_{3}}{\sqrt{3}}, \ldots, \frac{e_{m}}{\sqrt{m}}, \ldots, \frac{e_{m}}{\sqrt{m}}\right\}
$$

are both Parseval frames for $\mathbb{H}^{m}$. Zeros and repetitions are allowed.

We refer to [1] for an introduction to Hilbert space frame theory and applications.

The following are certain operators associated with frames:

The analysis operator for a frame $\Phi=\left\{\phi_{i}\right\}_{i=1}^{n}$ is defined as the operator $T: \mathbb{H}^{m} \rightarrow \ell_{2}^{n}$ to be

$$
T x=\left(\left\langle x, \phi_{1}\right\rangle,\left\langle x, \phi_{2}\right\rangle, \cdots,\left\langle x, \phi_{n}\right\rangle\right)=\sum_{i=1}^{n}\left\langle x, \phi_{i}\right\rangle e_{i}=\left\{\left\langle x, \phi_{i}\right\rangle\right\}_{i=1}^{n} \text {, for all } x \in \mathbb{H}^{m} \text {. }
$$

Here, $\left\{e_{i}\right\}_{i=1}^{n}$ is understood to be the natural orthonormal basis for $\ell_{2}^{n}$.

The synthesis operator $T^{*}$ which is the adjoint of the analysis operator $T$ for frame $\Phi=\left\{\phi_{i}\right\}_{i=1}^{n}$ is as:

$$
T^{*}\left(\left\{a_{i}\right\}_{i=1}^{n}\right)=\sum_{i=1}^{n} a_{i} \phi_{i}
$$

It is clear that $T^{*}\left(e_{i}\right)=\phi_{i}$.

Notice that we can represent it as a $m \times n$ matrix:

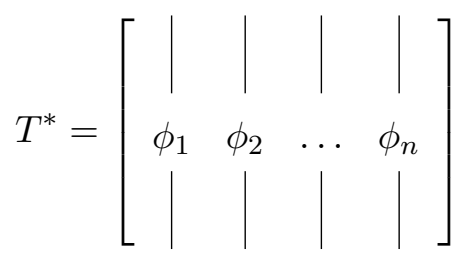

where the columns of $T^{*}$ represents the coefficients of the frame vectors with respect to $\left\{e_{i}\right\}_{i=1}^{n}$.

Notice that the columns of the synthesis operator in the matrix format, represent 
the coefficients of the frame vectors, then the square sum of each column represents the square norm of the frame vectors. Therefore a frame is equal norm if the square sum of all of the columns gives the same constant. Combining the synthesis operator and analysis operator creates frame operator:

The frame operator for the frame $\Phi$ is defined as $S=T^{*} T: \mathbb{H}^{m} \rightarrow \mathbb{H}^{m}$. That is,

$$
S x=T^{*} T(x)=\sum_{i=1}^{n}\left\langle x, \phi_{i}\right\rangle \phi_{i} \quad \text { for all } x \in \mathbb{H}^{m}
$$

and the Gram matrix is $\mathrm{G}_{T}=T^{*} T=\left(\left\langle\phi_{i}, \phi_{j}\right\rangle\right)_{i, j=1}^{n}$

Note that the frame operator $S$ is a positive, self-adjoint and invertible operator satisfying the operator inequality $A \cdot I \leq S \leq B \cdot I$, where $A$ and $B$ are the frame bounds and $I$ denotes the identity on $\mathbb{H}^{m}$.

Suppose $\mathrm{G}_{T}$ is the Gram matrix. If $\left\{\phi_{i}\right\}_{i=1}^{n}$ is a frame with frame operator $S$, having eigenvalues $\left\{\lambda_{i}\right\}_{i=1}^{m}$, then

$$
\sum_{i=1}^{n}\left\|\phi_{i}\right\|^{2}=\operatorname{Tr}\left(\mathrm{G}_{T}\right)=\operatorname{Tr}(S)=\sum_{j=1}^{m} \lambda_{j}
$$

So if $\left\{\phi_{i}\right\}_{i=1}^{n}$ is an equal norm Parseval frame then,

$$
\left\|\phi_{1}\right\|^{2}=\frac{1}{n} \sum_{i=1}^{n}\left\|x_{i}\right\|^{2}=\frac{m}{n}
$$

We can use the frame operator to reconstruct the vectors in the space. To be precise, any $x \in \mathbb{H}^{m}$ can be written as

$$
x=S S^{-1} x=S^{-1} S x=\sum_{i=1}^{n}\left\langle S^{-1} x, \phi_{i}\right\rangle \phi_{i}=\sum_{i=1}^{n}\left\langle x, S^{-1} \phi_{i}\right\rangle \phi_{i}
$$


And also,

$$
\begin{aligned}
\sum_{i=1}^{n}\left\langle x, S^{-1 / 2} \phi_{i}\right\rangle S^{-1 / 2} \phi_{i} & =S^{-1 / 2}\left(\sum_{i=1}^{n}\left\langle S^{-1 / 2} x, \phi_{i}\right\rangle \phi_{i}\right) \\
& =S^{-1 / 2}\left(S\left(S^{-1 / 2} x\right)\right)=x
\end{aligned}
$$

so $\left\{S^{-1 / 2} \phi_{i}\right\}_{i=1}^{n}$ is a Parseval frame. One reformulation of the frame definition is that the numerical range of $\mathrm{S}$, the set $\langle S x, x\rangle$ for all $\|x\|=1$, is an interval in the positive real numbers. Since $S$ is invertible, the family $\left\{S^{-1} \phi_{i}\right\}$ is also a frame for $\mathbb{H}$ called the canonical dual frame. It is also known that a frame is Parseval if and only if its frame operator is the identity operator.

The analysis operator of a Parseval frame is an isometry and the frame operator is the identity operator. Thus, if $\left\{\phi_{i}\right\}_{i=1}^{n}$ is a Parseval frame, it follows from equation (1.1) that

$$
x=\sum_{i=1}^{n}\left\langle x, \phi_{i}\right\rangle \phi_{i} \quad \text { for all } \quad x \in \mathbb{H}^{m}
$$

Let $T: \mathbb{H}^{m} \rightarrow \mathbb{H}^{m}$ be a linear operator. A nonzero vector $x \in \mathbb{H}^{m}$ is an eigenvector of $T$ with eigenvalue $\lambda$ if

$$
T x=\lambda x
$$

The operator $\mathrm{T}$ is diagonalizable if there exists an orthonormal basis for $\mathbb{H}^{m}$ consisting of eigenvectors for $T$. Notice that this definition is quiet different than the standard definition which says an operator is diagonalizable if there is some basis consisting of eigenvectors for $\mathrm{T}$. 
If $\mathrm{P}$ is a projection with respect to a frame in $\mathbb{H}^{m}$, we have:

$$
\langle P x, x\rangle=\left\langle P^{2} x, x\right\rangle=\langle P x, P x\rangle=\|P x\|^{2}
$$

so that $\mathrm{P}$ is a positive operator. If $P: \mathbb{H}^{m} \rightarrow W$ and $\operatorname{dim} W=m$, then $P$ has eigenvalue 1 with multiplicity $m$ and eigenvalue 0 of multiplicity $m-n$.

The next result, known as Naimark's theorem, characterizes Parseval frames in a finite dimensional Hilbert space. This theorem facilitates a way to construct Parseval frames, and crucially it is the only way to obtain Parseval frames. Later, we use this to obtain a classification of frames which satisfy norm retrieval.

Theorem 1.0.2 (Naimark's Theorem). [2] Let $\Phi=\left\{\phi_{i}\right\}_{i=1}^{n}$ be a frame for $\mathbb{H}^{m}$ with analysis operator $T$, let $\left\{e_{i}\right\}_{i=1}^{n}$ be the standard basis of $\ell_{2}^{n}$, and let $P: \ell_{2}^{n} \rightarrow \ell_{2}^{n}$ be the orthogonal projection onto range $T$. Then $\left\{\phi_{i}\right\}_{i=1}^{n}$ is a Perseval frame for $\mathbb{R}^{m}$ if and only if $P e_{i}=T \phi_{i}$ for all $i \in[n]$. Moreover, the Naimark Complement is $\left\{(I-P) e_{i}\right\}_{i=1}^{n}$.

Notice that the Naimark complement is only defined for Parseval frames and up to a unitary equivalence, that is, if $\left\{\phi_{i}\right\}_{i=1}^{n} \subset \mathbb{H}^{m}$ and $\left\{\psi_{i}\right\}_{i=1}^{n} \subset \mathbb{H}^{n-m}$ are Naimark complements, and $U$ and $V$ are unitary operators, then $\left\{U \phi_{i}\right\}_{i=1}^{n}$ and $\left\{V \psi_{i}\right\}_{i=1}^{n}$ are also Naimark complements.

With a slight abuse of notation we have:

Theorem 1.0.3. $\Phi=\left\{\phi_{i}\right\}_{i=1}^{n}$ is a Parseval frame for $\mathbb{H}^{m}$ if and only if there is an $n$ dimensional Hilbert space $\mathbb{H}^{m} \subset \mathcal{K}_{n}$ with an orthonormal basis $\left\{e_{i}\right\}_{i=1}^{n}$ such that the orthogonal projection $P: \mathcal{K}_{m} \rightarrow \mathbb{H}^{m}$ satisfies $P e_{i}=\phi_{i}$ for all $i \in[m]$. Moreover the Naimark complement of $\Phi$ is $\left\{(I-P) e_{i}\right\}_{i=1}^{n}$ 
Throughout this dissertation, an orthogonal projection or simply a projection is a self-adjoint projection.

In order to reconstruct an arbitrary frame without satisfying any strong properties like Parseval frame properties we can use a type of frames called dual frames:

Let $\Phi=\left\{\phi_{i}\right\}_{i=1}^{n}$ be a frame for $\mathbb{H}^{m}$. A frame $\left\{\psi_{i}\right\}_{i=1}^{m}$ is called a dual frame for $\Phi$ if for all $x \in \mathbb{H}^{m}$,

$$
x=\sum_{i=1}^{m}\left\langle x, \phi_{i}\right\rangle \psi_{i}
$$

What if the frame is linearly dependent? In this case the decomposition of a signal with respect to a frame is not unique. In order to have a unique decomposition without using strong conditions like orthogonality, we can use Riesz bases which provides uniqueness without using any specific property.

Definition 1.0.4. A frame $\left\{\phi_{i}\right\}_{i=1}^{n}$ in $\mathbb{H}^{m}$ is called Riesz basis for $\mathbb{H}^{m}$, if for all scalars $\left\{a_{i}\right\}_{i=1}^{n}$,

$$
A \sum_{i=1}^{n}\left|a_{i}\right|^{2} \leq\left\|\sum_{i=1}^{n} a_{i} \phi_{i}\right\|^{2} \leq B \sum_{i=1}^{n}\left|a_{i}\right|^{2}
$$

when $A$ and $B$ are lower and upper Riesz bounds.

A frame $\left\{\phi_{i}\right\}_{i=1}^{n}$ is said to be bounded if there is a constant $C>0$ such that for all $i \in[n],\left\|\phi_{i}\right\| \geq C$.

Two sets of vectors $\left\{\phi_{i}\right\}_{i=1}^{n}$ and $\left\{\psi_{i}\right\}_{i=1}^{n}$ are equivalent if the operator $L$ such that $L\left(\phi_{i}\right)=\psi_{i}$ is a well defined bounded invertible operator. 
One of the variations of frames is to scale its frame vectors. If by scaling a frame we get a Parseval frame then we can say that the frame was scalable. Notice that the scaling of frames is related to the notion of signed frames, weighted frames and controlled frames (see [26], [27]). Here is the definition of scalable frames:

Definition 1.0.5. A frame $\Phi=\left\{\phi_{i}\right\}_{i \in \mathrm{I}}$ for $\mathbb{H}^{m}$ is called scalable if there exist scalars $c_{i} \geq 0, i \in \mathrm{I}$, such that $\left\{c_{i} \phi_{i}\right\}_{i \in \mathrm{I}}$ is a Parseval frame. Also we will have:

- If $c_{i}>0$ for all $i \in \mathrm{I}$, then $\Phi$ is called positively scalable.

- If there exists $\delta>0$, such that $c_{i} \geq \delta$ for all $i \in \mathrm{I}$, then $\Phi$ is called strictly scalable.

For finite frames, positive and strict scalability are the same, and each scaling $\left\{c_{i} \phi_{i}\right\}_{i \in \mathrm{I}}$ of a finite frame $\left\{c_{i}\right\}_{i \in \mathrm{I}}$ with positive scalars $c_{i}$ is again a frame. In the infinite dimensional situation this might not be the case. However, if there exist $m_{1}, m_{2}>0$ such that $m_{1} \leq c_{i} \leq m_{2}$ holds for all $i \in \mathrm{I}$, then also $\left\{c_{i} \phi_{i}\right\}_{i \in \mathrm{I}}$ is a frame. 


\section{Chapter 2}

\section{Phase Retrieval and Norm Retrieval}

In this section, we will give the formal definitions of phase retrieval, phaseless reconstruction, and norm retrieval.

\subsubsection{Start of Phase Retrieval}

Phase retrieval contains methods for recovering the phase of a signal given its intensity measurements from a redundant linear system. Recovering signals when there is partial loss of information is a significant challenge. Partial loss of phase information occurs in application areas such as speech recognition, and optics applications such as X-ray crystallography, and there is a need to do phase retrieval efficiently. X-ray crystallography is the most well-known application of phase retrieval, in which physicists expose a crystal to X-rays in order to determine its molecular geometry; First the X-rays are taken repeatedly as the crystal rotates. When the X-ray beam hits the atom, it gets diffracted in many patterns. Diffraction patterns will correspond to the modulus-squared of the object's Fourier transform. 
In the 1950's this was the technology that led to the discovery that DNA is a double helix which ultimately won Watson and Crick a Nobel Prize.

In recent years, phase retrieval has become foundational to the related application of coherent diffractive imaging in which one attempts to image nanoscale objects based on the diffraction pattern measured after the object is exposed to powerful X-ray beams. In speech recognition technology, reconstruction of a signal using noisy phase or its estimation can be a critical problem needing phase retrieval.

In the discrete setting, these measurements correspond to the magnitude of the inner products with the given frame vectors, or $\left|\left\langle x, \phi_{i}\right\rangle\right|$. Measurements of this type have an inherent ambiguity, since $\left|\left\langle x, \phi_{i}\right\rangle\right|=\left|\left\langle e^{i \theta} x, \phi_{i}\right\rangle\right|$ for all $\theta \in \mathbb{R}^{n}$. By phase, we are referring to the unimodular portion of the polar decomposition of $x$ i.e $\left|e^{i \theta}\right|$.

\subsubsection{Main Problems and Definitions}

Phase retrieval has been defined for vectors as well as for projections and in general deals with recovering the phase of a signal given its intensity measurements from a redundant linear system. Phase retrieval by projections, where the signal is projected onto some lower dimensional subspaces and has to be recovered from the norms of the projections of the vectors onto the subspaces, appears in real life problems such as crystal twinning [17]. We refer the reader to [3] for a detailed study of phase retrieval by projections.

Another related problem is that of phaseless reconstruction, where the unknown signal is reconstructed from the intensity measurements. Recently, the two terms phase retrieval and phaseless reconstruction were used interchangeably. However, it is not clear from their respective definitions how these two are equivalent. Recently, in $[15]$ the authors proved the equivalence of phase retrieval and phaseless reconstruction in the real as well as in complex case. 
For consistency in this dissertation, we restrict ourselves to provide results regarding phase retrieval. Further, a weaker notion of phase retrieval and phaseless reconstruction [5] will be introduced in the next chapter.

In phase retrieval, one of the main questions is to figure out how to design measurement vectors $\left\{\phi_{i}\right\}_{i=1}^{n}$ so that any vector $x$ can be determined from $\left\{\left|\left\langle x, \phi_{i}\right\rangle\right|^{2}\right\}_{i=1}^{n}$ ? There are two main approaches to this problem of phase retrieval. The first one is to restrict the problem to a subclass of signals on which the intensity measurements become injective which is known as classical phase retrieval. The other approach is to use a larger family of measurements so that the intensity measurements map any signal injectively.

Definition 2.0.1. (Classical Phase Retrieval) A family of vectors $\left\{\phi_{i}\right\}_{i=1}^{n}$ in $\mathbb{H}^{m}$ allows phase retrieval if for all $x, y \in \mathbb{H}^{m}$ satisfying $\left|\left\langle x, \phi_{i}\right\rangle\right|=\left|\left\langle y, \phi_{i}\right\rangle\right|$ for all $i \in[n]$, then $x=$ cy where $c= \pm 1$ in $\mathbb{R}^{m}$ (for $\mathbb{C}^{m}, c$ is in the complex unit circle)

The second approach in phase retrieval was first defined by Balan, Casazza and Edidin [7] where they examine injectivity of intensity measurements for finite dimensional Hilbert spaces. The definition of phase retrieval along with other definitions that we mostly use throughout this dissertation is as follows:

Definition 2.0.2. Let $\Phi=\left\{\phi_{i}\right\}_{i=1}^{n}$ be a family of vectors $\in \mathbb{H}^{m}$. For every non-zero $x, y \in \mathbb{H}^{m}$ satisfying:

$$
\left|\left\langle x, \phi_{i}\right\rangle\right|=\left|\left\langle y, \phi_{i}\right\rangle\right| \quad \text { for all } i \in[n]
$$

1. If this implies there exist $|\theta|=1$ such that $x_{i}=\theta y_{i}$ then $\Phi$ yields phase retrieval with respect to an orthonormal basis $\left\{e_{i}\right\}_{i=1}^{m}$ when $x_{i}=\left\langle x, e_{i}\right\rangle$. 
2. A family of projections $\left\{P_{i}\right\}_{i=1}^{n}$ on $\mathbb{H}^{m}$ does phase retrieval if for all $x, y \in \mathbb{H}^{m}$, $\left\|P_{i} x\right\|^{2}=\left\|P_{i} y\right\|^{2}$ implies $x=\theta y$ for some $|\theta|=1$.

3. phaseless reconstruction if there is a $|\theta|=1$ such that $x=\theta y$.

4. If this implies $\|x\|=\|y\|$ then $\Phi$ yields norm retrieval.

We note that tight frames $\left\{\phi_{i}\right\}_{i=1}^{m}$ for $\mathbb{H}^{m}$ do norm retrieval. Indeed, if

$$
\left|\left\langle x, \phi_{i}\right\rangle\right|=\left\langle y, \phi_{i}\right\rangle \mid \quad \text { for all } i \in[m]
$$

then

$$
A\|x\|^{2}=\sum_{i=1}^{m}\left|\left\langle x, \phi_{i}\right\rangle\right|^{2}=\sum_{i=1}^{m}\left|\left\langle y, \phi_{i}\right\rangle\right|^{2}=A\|y\|^{2}
$$

Orthonormal bases fail to do phase retrieval, since in any given orthonormal basis, the corresponding coefficients of a vector are unique. Phase retrieval in $\mathbb{R}^{m}$ is classified in terms of a fundamental result called the complement property.

Definition 2.0.3. [7] A frame $\Phi=\left\{\phi_{i}\right\}_{i=1}^{n}$ in $\mathbb{H}^{m}$ satisfies the complement property if for all subsets $I \subset[n]$, either $\operatorname{span}\left\{\phi_{i}\right\}_{i \in I}=\mathbb{H}^{m}$ or $\operatorname{span}\left\{\phi_{i}\right\}_{i \in I^{c}}=\mathbb{H}^{m}$.

Notice that phase retrieval is impossible without injective intensity measurements. In [7] the authors first introduced the complement property to analyze injectivity in the classical phase retrieval settings. A fundamental result from [7] is:

Theorem 2.0.4 ([7]). If $\Phi$ does phase retrieval then it has complement property. In a real Hilbert space, if $\Phi$ has complement property then it does phase retrieval.

This theorem classifies phase retrievable frames in the real setting. It follows that if $\Phi=\left\{\phi_{i}\right\}_{i=1}^{n}$ does phase retrieval in $\mathbb{R}^{m}$ then $n \geq 2 m-1$. It is also known [7] that $\Phi$ never does phase retrieval when $n<2 m-1$. 
We can observe this by choosing $n=2 m-2$. In this case, it is possible to partition the vectors into two sets of cardinality $m-1$. It is clear that complement property fails since there are not enough vectors either of the two partitions to span the $m$-dimensional space.

Notice that complement property is a necessary condition to satisfy phase retrieval in the complex case but the question of finding the minimum number of measurements necessary to satisfy phase retrieval remains open. A counter example in [25] disproves the conjecture which says the minimum number of vectors necessary to satisfy phase retrieval in $\mathbb{C}^{m}$ is $4 m-4$.

Full spark is another important notion of vectors in frame theory. A formal definition is given below:

Definition 2.0.5. Given a family of vectors $\Phi=\left\{\phi_{i}\right\}_{i=1}^{n}$ in $\mathbb{H}^{m}$, the spark of $\Phi$ is defined as the cardinality of the smallest linearly dependent subset of $\Phi$. When $\operatorname{spark}(\Phi)=m+1$, every subset of size $m$ is linearly independent, and in that case, $\Phi$ is said to be full spark.

From the definition, it follows that full spark frames with $n \geq 2 m-1$ have the complement property and hence do phase retrieval. Moreover, if $n=2 m-1$ then the complement property implies full spark. Note that full spark is a much stronger property than the complement property. The notion of spark is the measure of how resilient a frame is against erasures, so full spark is a desired property of a frame. In general, it is not necessary for a frame to be full spark in order to yield phase retrieval; as long as our frame contains a full spark subset of $2 m-1$ vectors, it will do phase retrieval. However, if the frame contains exactly $2 m-1$ vectors, then clearly it does phase retrieval if and only if it is full spark. 


\subsubsection{Subspaces and Projections}

In this section we first state the properties of subspaces satisfying phase retrieval which is based on the work in [3] and later we will provide the detailed characterization for subspaces allowing norm retrieval. The definition of phase retrieval property for subspaces is as follows:

Definition 2.0.6. Let $\left\{W_{i}\right\}_{i=1}^{n}$ be a collection of subspaces of $\mathbb{H}^{m}$ and let $\left\{P_{i}\right\}_{i=1}^{n}$ be the orthogonal projections of the subspaces respectively. Then we say $\left\{W_{i}\right\}_{i=1}^{n}$ on $\left\{P_{i}\right\}_{i=1}^{n}$ does phase retrieval if for all $x, y \in \mathbb{H}^{m}$ satisfying

$$
\left\|P_{i} x\right\|=\left\|P_{i} y\right\| \quad \text { for all } i \in[n]
$$

then $x=$ cy for some scalar $|c|=1$.

Characterizing subspaces that allow phase retrieval has been studied in [3]. The idea is to use self adjoint operators when the norms of projections was chosen as the measurements.

The family of $m \times m$ self-adjoint real matrices with $\mathbb{H}^{m \times m}$ is a $m(m+1) / 2$ dimensional vector space. For a family of subspaces $\left\{W_{i}\right\}_{i=1}^{n}$ in $\mathbb{R}^{m}$ with corresponding projections $P_{i}$, define the operator $T: \mathbb{H}^{m \times m} \rightarrow \mathbb{R}^{m}$ as $T A(n)=\left\langle A, P_{i}\right\rangle_{H S}$ where $\langle,\rangle_{H S}$ is the Hilbert-Schmidt inner product. Let $\left\{\phi_{i, j}\right\}_{j=1}^{J_{i}}$ be an orthonormal basis for $W_{i}$. Notice for any $x \in \mathbb{R}^{m}$,

$$
T\left(x x^{*}\right)(i)=\left\langle x x^{*}, P_{i}\right\rangle=\operatorname{Tr}\left(x x^{*} P_{i}\right)=\operatorname{Tr}\left(x x^{*} \sum_{j=1}^{J_{i}} \phi_{i, j} \phi_{i, j}^{*}\right)
$$

Since the trace has a cyclic property,

$$
\operatorname{Tr}\left(x x^{*} \sum_{j=1}^{J_{i}} \phi_{i, j} \phi_{i, j}^{*}\right)=\sum_{j=1}^{J_{i}} \phi_{i, j}^{*} x x^{*} \phi_{i, j}=\sum_{j=1}^{J_{i}}\left|\left\langle x, \phi_{i, j}\right\rangle\right|=\left\|P_{i} x\right\|^{2}
$$


Therefore $T\left(x x^{*}\right)(i)=\left\|P_{i} x\right\|^{2}$

The next lemma gives a characterization for when subspaces allow phase retrieval in the classical phase retrieval.

Lemma 2.0.7. [8] Let $\Phi=\left\{\phi_{i}\right\}_{i=1}^{n}$ be a family of vectors in $\mathbb{R}^{m}$. Then $\Phi$ allows phase retrieval if and only if the null space of $G: \mathbb{H}^{m \times m} \rightarrow \mathbb{R}^{n}$ given by $G A(n)=\left\langle A, \phi_{i} \phi_{i}^{*}\right\rangle_{H S}$ does not contain a matrix of rank 1 or 2 .

In [3] the authors generalized this result to projections of arbitrary ranks, it turns out that the characterization is identical.

Corollary 2.0.8. Given subspaces $\left\{W_{i}\right\}_{i=1}^{n}$ in $\mathbb{R}^{m}$ with corresponding projections $P_{i}$, $\left\{W_{i}\right\}_{i=1}^{n}$ allows phase retrieval if and only if there are no matrices of rank 1 or 2 in the null space of $T$.

Notice that $2 m-1$ is the minimum number of vectors required to do phase retrieval, therefore we would hope to find the minimum number of subspaces required to do phase retrieval by just analyzing the similarities between the lemma and the corollary. The main issue here is that the space of rank 1 and rank 2 operators do not form a subspace in $\mathbb{H}^{m \times m}$, and null spaces of $T$ and $G$ may (or may not) intersect this space in fundamentally different ways. The minimal number $2 m-1$ for phase retrieval with vectors since this is the least number of vectors which satisfy the complement property in $\mathbb{R}^{m}$. We do not have complement property for subspaces, therefore it makes it harder to find the minimum number of subspaces satisfying phase retrieval.

The following results from [3] will provide the characterization for subspaces doing phase retrieval which is based on lemma 2.0.7 and corollary 2.0.8 and we state it without the proof.

Corollary 2.0.9. [3] Let $\left\{W_{i}\right\}_{i=1}^{n}$ be subspaces of $\mathbb{H}^{m}$ doing phase retrieval. For every orthonormal basis $\left\{\phi_{i, j}\right\}_{j=1}^{J_{i}}$ of $\left\{W_{i}\right\}$, the set $_{15} \Phi=\left\{\phi_{i, j}\right\}_{i=1, j=1}^{n, J_{i}}$ allows phase retrieval 
in $\mathbb{R}^{m}$.

Lemma 2.0.10. [3] Let $P$ be the orthogonal projection onto an $n$-dimensional subspace $W \subset \mathbb{R}^{m}$. Given $x, y \in \mathbb{R}^{m}$ the following are equivalent:

1. $\|P x\|=\|P y\|$

2. There exists an orthonormal basis $\left\{\phi_{i}\right\}_{i=1}^{n}$ for $W$ such that $\left|\left\langle x, \phi_{i}\right\rangle\right|=\left|\left\langle y, \phi_{i}\right\rangle\right|$ for all $i \in[n]$

Combining the previous corollary and lemma, we can now see the characterization of subspaces allowing phase retrieval in $\mathbb{R}^{m}$ in terms of the complement property.

Theorem 2.0.11. [3] Let $\left\{W_{i}\right\}_{i=1}^{n}$ be subspaces of $\mathbb{R}^{m}$. The following are equivalent:

1. $\left\{W_{i}\right\}_{i=1}^{n}$ allows phase retrieval for $\mathbb{R}^{m}$

2. For every orthonormal basis $\left\{\phi_{i, j}\right\}_{j=1}^{J_{i}}$ of $\left\{W_{i}\right\}$ the set $\left\{\phi_{i, j}\right\}_{j=1}^{J_{i}}$ allows phase retrieval in $\mathbb{R}^{m}$ and thus has the complement property.

It is interesting to observe that we can also use theorem 2.0.11 for subspaces and not only vectors. Take each $W_{i}$ and split this subspace into orthogonal subspaces which span $W_{i}$. It is important to note that we used orthogonal bases in the previous theorem since complement property is very convenient to apply.

Corollary 2.0.12. Let $\left\{W_{i}\right\}_{i=1}^{n}$ be subspaces of $\mathbb{R}^{m}$. The following are equivalent:

1. $\left\{W_{i}\right\}_{i=1}^{n}$ allow phase retrieval for $\mathbb{R}^{m}$

2. For every choice of orthogonal subspaces $\left\{Z_{i, j}\right\}_{j=1}^{J_{i}}$ where $\oplus_{j=1}^{J_{i}} Z_{i, j}=W_{i}$ the subspaces $\left\{Z_{i, j}\right\}_{i=1, j=1}^{n, J_{i}}$ allow phase retrieval in $\mathbb{R}^{m}$ 


\subsubsection{Norm Retrieval}

One of the major questions in this context is about complements of the subspaces; If $\left\{W_{i}\right\}_{i=1}^{n}$ do phase retrieval, do $\left\{W_{i}^{\perp}\right\}_{i=1}^{n}$ yield phase retrieval? In other words, if we move to the complements of our subspaces, do we still have phase retrieval property? It is shown in [3] that this is not true in general. Norm retrieval is the property needed to pass phase retrieval to orthogonal complements. In this section, we will see some properties of norm retrieval along with certain related results, and pertinent examples.

Definition 2.0.13. Let $\left\{W_{i}\right\}_{i=1}^{n}$ be a collection of subspaces in $\mathbb{H}^{m}$ and let $\left\{P_{i}\right\}_{i=1}^{n}$ be the orthogonal projections onto each of these subspaces. We say that $\left\{W_{i}\right\}_{i=1}^{n}$ (or $\left.\left\{P_{i}\right\}_{i=1}^{n}\right)$ yields norm retrieval if for all $x, y \in \mathbb{H}^{m}$ satisfying $\left\|P_{i} x\right\|=\left\|P_{i} y\right\|$ for all $i=1,2, \ldots, n$ then $\|x\|=\|y\|$.

In particular, a set of vectors $\left\{\phi_{i}\right\}_{i=1}^{n}$ in $\mathbb{H}^{m}$ does norm retrieval, if for $x, y \in \mathbb{H}^{m}$ satisfying $\left|\left\langle x, \phi_{i}\right\rangle\right|=\left|\left\langle y, \phi_{i}\right\rangle\right|$ for all $i \in[n]$ then $\|x\|=\|y\|$.

Remark 2.0.14. It is immediate that a family of vectors allowing phase retrieval, satisfies norm retrieval. However the converse might not hold in general since we might have too few vectors to span the space.

An obvious choice of vectors which do norm retrieval are orthonormal bases. For example, let $\left\{e_{i}\right\}_{i=1}^{m}$ be an orthonormal basis in $\mathbb{H}^{m}$. Now, for $x \in \mathbb{H}^{m},\left|\left\langle x, \phi_{i}\right\rangle\right|=$ $\left|\left\langle x, e_{i}\right\rangle\right|=\left|x_{i}\right|$. Thus

$$
\sum_{i=1}^{m}\left|\left\langle x, \phi_{i}\right\rangle\right|^{2}=\sum_{i=1}^{m}\left|x_{i}\right|^{2}=\|x\|^{2} .
$$

The following theorem provides a sufficient condition under which the subspaces spanned by the canonical basis vectors allow norm retrieval. 
Theorem 2.0.15. Let $\left\{e_{i}\right\}_{i=1}^{m}$ be an orthonormal basis in $\mathbb{H}^{m}$. Let $\left\{W_{j}\right\}_{j=1}^{k}$ be subspaces of $\mathbb{H}^{m}$ where each $W_{j}=\operatorname{span}\left\{e_{i}\right\}_{i \in I_{j}}, I_{j} \subseteq[m]$. If there exists $M$ such that for all $j,\left|\left\{j: e_{i} \in W_{j}\right\}\right|=M$, then $\left\{W_{j}\right\}_{j=1}^{k}$ does norm retrieval.

Proof. Let $P_{j}$ be orthogonal projections onto $W_{j}$, for all $j$. Now, by assumption, we have

$$
\sum_{j=1}^{k}\left\|P_{j} x\right\|^{2}=\sum_{j=1}^{k} \sum_{i \in I_{j}}\left|\left\langle x, e_{i}\right\rangle\right|^{2}=M \sum_{j=1}^{m}\left|\left\langle x, e_{j}\right\rangle\right|^{2}=M\|x\|^{2}
$$

It is easy to see that tight frames do norm retrieval:

Theorem 2.0.16. Tight frames do norm retrieval.

Proof. Let $\left\{\phi_{i}\right\}_{i=1}^{n}$ in $\mathbb{H}^{m}$ be an A-tight frame. If

$$
\left|\left\langle x, \phi_{i}\right\rangle\right|=\left\langle y, \phi_{i}\right\rangle \mid, \text { for all } i \in[n]
$$

then

$$
A\|x\|^{2}=\sum_{i=1}^{n}\left|\left\langle x, \phi_{i}\right\rangle\right|^{2}=\sum_{i=1}^{n}\left|\left\langle y, \phi_{i}\right\rangle\right|^{2}=A\|y\|^{2}
$$

which proves that $\|x\|=\|y\|$ which satisfies norm retrieval.

It is important to characterize different subspaces that do norm retrieval and see if the union of subspaces also carry the same properties as each one. Observe that if $\left\{\phi_{i}\right\}_{i=1}^{n} \in \mathbb{H}^{m}$ allow norm retrieval so does $\left\{\phi_{i}\right\}_{i=1}^{n} \cup\left\{\psi_{j}\right\}_{j=1}^{k}$ for any $\left\{\psi_{j}\right\} \in \mathbb{H}^{m}$. This is generalized in the following proposition. 
Proposition 2.0.17. If the family of projections with respect to an orthonormal basis, $\left\{P_{i}\right\}_{i=1}^{n}$ allow norm retrieval, then so does $\left\{P_{i}\right\}_{i=1}^{n} \cup\left\{Q_{i}\right\}_{i=1}^{k}$ for any projections $\left\{Q_{i}\right\}$. In particular, if a family of vectors $\Phi=\left\{\phi_{i}\right\}_{i=1}^{n}$ contains an orthonormal basis, then it does norm retrieval.

Moreover, in this case, $\left\{\phi_{i}^{\perp}\right\}_{i=1}^{n}$ also allows norm retrieval.

Proof. Let $\left\{e_{i}\right\}_{i=1}^{m}$ be an orthonormal basis for $\mathbb{H}^{m}$ and let $\left\{P_{i}\right\}_{i=1}^{m}$ be the respective projection onto $\phi_{i}{ }^{\perp}$, for each $i \in[m]$. Given $x \in \mathbb{H}^{m}$, we have

$$
\sum_{i=1}^{m}\left\|P_{i} x\right\|^{2}=\sum_{i=1}^{m} \sum_{j \neq i}\left|\left\langle x, e_{j}\right\rangle\right|^{2}=(m-1) \sum_{i=1}^{m}\left|\left\langle x, e_{j}\right\rangle\right|^{2}=(m-1)\|x\|^{2}
$$

The above proposition does not hold if the number of hyperplanes is strictly less than $m$. This is proved in the next theorem.

Theorem 2.0.18. If $\left\{\phi_{i}\right\}_{i=1}^{m}$ is an orthonormal basis for $\mathbb{R}^{m}$ then $\left\{W_{i}\right\}_{i \in I}$ where $\left\{W_{i}\right\}=\left\{\phi_{i}\right\}^{\perp}$ cannot do norm retrieval for $I \subseteq[m-1]$.

Proof. Consider the family of subspaces $\left\{W_{i}\right\}_{i=1}^{m-1}$, without loss of generality suppose $m>2$. Now suppose $x$ and $y$ are as follows:

$$
x=\sum_{i=1}^{m} \phi_{i} \quad \text { and } \quad y=\sqrt{\frac{m-1}{m-2}} \sum_{i=1}^{m-1} \phi_{i}
$$

now,

$$
\left\|P_{j} x\right\|^{2}=\sum_{\substack{i=1 \\ i \neq j}}^{m}\left|\left\langle x, \phi_{i}\right\rangle\right|^{2}=m-1
$$

and,

$$
\left\|P_{j} y\right\|^{2}=\frac{m-1}{m-2} \sum_{\substack{i=1 \\ i \neq j}}^{m-1}\left|\left\langle x, \phi_{i}\right\rangle\right|^{2}=m-1
$$


Therefore, $\left\|P_{j} x\right\|^{2}=\left\|P_{j} y\right\|^{2}$. However,

$$
\|x\|^{2}=m \quad \text { and } \quad\|y\|^{2}=\frac{(m-1)^{2}}{m-2}
$$

which shows that norm retrieval is not possible.

Now if we consider the collection $\left\{W_{i}\right\}_{i=1}^{m}$, then given $x=\left(x_{1}, x_{2}, \ldots, x_{m}\right)$ and $y=\left(y_{1}, y_{2}, \ldots, y_{m}\right)$ such that $\left\|P_{i} x\right\|^{2}=\sum_{j \neq i}\left\|x_{j}\right\|^{2}=\left\|P_{i} y\right\|^{2}=\sum_{j \neq i}\left\|y_{j}\right\|^{2}$. Then we have

$$
(m-1)\|x\|^{2}=\sum_{i=1}^{m}\left\|P_{i} x\right\|^{2}=\sum_{i=1}^{m}\left\|P_{i} y\right\|^{2}=(m-1)\|y\|^{2}
$$

Therefore the above theorem holds only if we have at least $\mathrm{m}$ hyperplanes.

We will make the above result stronger by excluding the orthogonality from its properties in the next proposition, but first we need the following lemma:

Lemma 2.0.19. If $\left\{\phi_{i}\right\}_{i=1}^{m}$ are linearly independent vectors in $\mathbb{R}^{m}$, then $\forall c>0$ there is a vector $x \in \mathbb{R}^{m}$ satisfying:

$$
\left|\left\langle x, \phi_{i}\right\rangle\right|=c \neq 0, \text { for all } i \in[m] .
$$

Proof. Proof by induction on $m$.

Case $m=2$ is obvious.

Now assume the equality holds for $m-1$. Given a family of vectors $\left\{\phi_{i}\right\}_{i=1}^{m}$, we can find $x$ such that $x \in \operatorname{span}\left\{\phi_{i}\right\}_{i=1}^{m-1}$ and satisfying

$$
\left|\left\langle x, \phi_{i}\right\rangle\right|=c \neq 0, \text { for all } i \in[m-1]
$$


Choose $y \perp$ span $\left\{\phi_{i}\right\}_{i=1}^{m-1}$ and note that linear independence of the $\left\{\phi_{i}\right\}$ implies

$$
\left|\left\langle y, \phi_{m}\right\rangle\right| \neq 0
$$

Consider $x+\lambda y$. For $i \in[m-1]$,

$$
\begin{aligned}
\left|\left\langle x+\lambda y, \phi_{i}\right\rangle\right| & =\left|\left\langle x, \phi_{i}\right\rangle+\lambda\left\langle y, \phi_{i}\right\rangle\right| \\
& =\left|\left\langle y, \phi_{i}\right\rangle\right| \\
& =c
\end{aligned}
$$

Also,

$$
\left\langle x+\lambda y, \phi_{m}\right\rangle=\left\langle x, \phi_{m}\right\rangle+\lambda\left\langle y, \phi_{m}\right\rangle
$$

As $\lambda$ varies from $-\infty$ to $+\infty$, the right hand side varies from $-\infty$ to $+\infty$ and for some $\lambda$, we have

$$
\left|\left\langle x, \phi_{m}\right\rangle+\lambda\left\langle y, \phi_{m}\right\rangle\right|=c
$$

Now in proof of the following proposition we will use the previous lemma:

Proposition 2.0.20. If $\left\{\phi_{i}\right\}_{i=1}^{m-1} \in \mathbb{R}^{m}$ are linearly independent and unit norm and $\left\{W_{i}\right\}=\left\{\phi_{i}\right\}^{\perp}$, for all $i \in[m-1]$, then $\left\{W_{i}\right\}_{i=1}^{m-1}$ cannot do norm retrieval.

Proof. Suppose $\left\{P_{i}\right\}_{i=1}^{m-1}$ is a family of projections onto the respective subspaces $\left\{W_{i}\right\}_{i=1}^{m-1}$ and choose a vector $x$ with $\|x\|=1$ so that:

$$
x \in \bigcap_{i=1}^{m-1} W_{i}
$$

By previous lemma 2.0.19, pick any $c>0$ then there is a vector $v \in \operatorname{span}\left\{\phi_{i}\right\}_{i=1}^{m-1}$ so that $\left|\left\langle v, \phi_{i}\right\rangle\right|=c \neq 0$, for all $i \in[m-1]$. 
In particular, we may scale $v$ and $c$ simultaneously such that $\|v\|=1$ and it follows that $0<c<1$.

Let $y=\lambda x+\mu v$ for some $\lambda$ and $\mu$, where $\lambda^{2}+\left(1-c^{2}\right) \mu^{2}=1$. Note that since $x \in\left\{\phi_{i}\right\}^{\perp}$ then $x \perp \phi_{i}$ for all $i \in[m-1]$, implies that $v \perp x$, and so:

$$
\|y\|^{2}=\lambda^{2}+\mu^{2} \neq 1
$$

Now, for all $i \in[m-1]$,

$$
\begin{aligned}
\left\|P_{i} y\right\|^{2} & =\|y\|^{2}-\left|\left\langle y, \phi_{i}\right\rangle\right|^{2} \\
& =\lambda^{2}+\mu^{2}-\mu^{2} c^{2} \\
& =\lambda^{2}+\left(1-c^{2}\right) \mu^{2} \\
& =1 \\
& =\|x\|^{2} \\
& =\left\|P_{i} x\right\|^{2} .
\end{aligned}
$$

But $\|x\|^{2}=1$ while $\|y\|^{2} \neq 1$, and so norm retrieval fails.

Previously we proved that we can not do norm retrieval with less than $m$ vectors in $\mathbb{H}^{m}$. However, in the following theorem, we show that three proper subspaces of codimension one can do norm retrieval in $\mathbb{R}^{m}$.

Theorem 2.0.21. In $\mathbb{R}^{m}$ three proper subspaces of codimension one can do norm retrieval.

Proof. Let $\left\{e_{i}\right\}_{i=1}^{m}$ be an orthonormal basis for $\mathbb{R}^{m}$. Let

$$
\phi_{1}=e_{1} \quad \phi_{2}=e_{2} \quad \phi_{3}=\left(e_{1}-e_{2}\right) / \sqrt{2}
$$


Claim: $\left\{\phi_{i}^{\perp}\right\}_{i=1}^{3}$ does norm retrieval.

Let $P_{i}$ be the orthogonal projection onto $\phi_{i}^{\perp}$ and choose $x=\left(a_{1}, \ldots, a_{m}\right)$.

We then have,

$$
\begin{aligned}
\left\|P_{1} x\right\|^{2} & =a_{2}^{2}+\sum_{k=3}^{m} a_{k}{ }^{2} \\
\left\|P_{2} x\right\|^{2} & =a_{1}^{2}+\sum_{k=3}^{m} a_{k}{ }^{2} \\
\left\|P_{3} x\right\|^{2} & =\left(\frac{a_{1}+a_{2}}{\sqrt{2}}\right)^{2}+\sum_{k=3}^{m} a_{k}{ }^{2} \\
& =\frac{a_{1}^{2}+2 a_{1} a_{2}+a_{2}^{2}}{2}+\sum_{k=3}^{m}{a_{k}}^{2}
\end{aligned}
$$

We then have 2 cases:

- Case 1: If $a_{1}=0$ or $a_{2}=0$, we know that $\|x\|^{2}=\left\|P_{1} x\right\|^{2}$ or $\|x\|^{2}=\left\|P_{2} x\right\|^{2}$ respectively.

- Case 2: Assume both $a_{1} \neq 0$ and $a_{2} \neq 0$. Therefore both of the equalities below hold:

$$
\begin{aligned}
& -\frac{\left(a_{1}+a_{2}\right)^{2}}{2} \cdot \frac{1}{a_{2}{ }^{2}}\left\|P_{1} x\right\|^{2}+\left\|P_{3} x\right\|^{2}=c \sum_{k=3}^{m} a_{k}{ }^{2} \\
& -\frac{\left(a_{1}+a_{2}\right)^{2}}{2} \cdot \frac{1}{a_{1}{ }^{2}}\left\|P_{2} x\right\|^{2}+\left\|P_{3} x\right\|^{2}=d \sum_{k=3}^{m} a_{k}{ }^{2}
\end{aligned}
$$

where

$$
c=-\frac{\left(a_{1}+a_{2}\right)^{2}}{2 a_{2}^{2}}+1 \quad \text { and } \quad d=-\frac{\left(a_{1}+a_{2}\right)^{2}}{2 a_{1}^{2}}+1
$$

If either $c$ or $d$ is nonzero, we can express $\|x\|^{2}$ as a linear combination of $\left\|P_{1} x\right\|^{2}$, $\left\|P_{2} x\right\|^{2}$ and $\left\|P_{3} x\right\|^{2}$ and this completes the proof.

Now, suppose $c=d=0$ :

If $c=0$, then $\left(a_{1}+a_{2}\right)^{2}=2 a_{2}{ }^{2}$ and if $d=0$, then $\left(a_{1}+a_{2}\right)^{2}=2 a_{1}{ }^{2}$. This 
implies that

$$
2\left(a_{1}+a_{2}\right)^{2}=2 a_{1}^{2}+2 a_{2}^{2}
$$

which holds only if either $a_{1}$ or $a_{2}$ or both is zero which is not possible since it contradicts our assumption.

The previous theorem gets even more interesting when we look at $\mathbb{R}^{3}$. It implies that in $\mathbb{R}^{3}$, two 2-dimensional subspaces cannot do norm retrieval but three 2-dimensional subspaces can do norm retrieval.

It is important to understand the relationship between the subspaces that allow norm retrieval and the complements of subspaces considering the dimensions of them.

Proposition 2.0.22. For every $k \leq m$, there exist subspaces $\left\{W_{i}\right\}_{i=1}^{k+1}$ in $\mathbb{H}^{m}$ which do norm retrieval and $\left\{W_{i}^{\perp}\right\}_{i=1}^{k+1}$ span a $k$-dimensional space.

Proof. Let $\left\{e_{i}\right\}_{i=1}^{m}$ be an orthonormal basis for $\mathbb{H}^{m}$. Let $W_{1}=\operatorname{span}\left\{e_{i}\right\}_{i=1}^{m-k}$ and $W_{i}=\operatorname{span}\left\{W_{1}, e_{m-k+i-1}\right\}$ for all $2 \leq i \leq k+1$.

Suppose for a vector $x$ such that $x=\sum_{j=1}^{m} a_{j} e_{j}$, then:

$$
\left\|P_{1} x\right\|^{2}=\sum_{j=1}^{m-k}\left|a_{j}\right|^{2}
$$

and

$$
\left\|P_{i} x\right\|^{2}=\sum_{j=1}^{m-k}\left|a_{j}\right|^{2}+\left|a_{m-k+i-1}\right|^{2} \quad \text { for } \quad 2 \leq i \leq k+1
$$

therefore

$$
\|x\|^{2}=\sum_{i=2}^{k+1}\left\|P_{i} x\right\|^{2}-(k-1)\left\|P_{1} x\right\|^{2}
$$


Since $W_{i}{ }^{\perp} \subseteq W_{1}^{\perp}$ for all $i \in[k+1]$, it's clear that $\left\{W_{i}^{\perp}\right\}_{i=1}^{k+1}$ is spanned by $\left\{e_{i}\right\}_{i=m-k+1}^{m}$, which has dimension $k$.

The following proposition shows a relationship between subspaces doing norm retrieval and the sum of the dimensions of the subspaces. Notice that we are looking for conditions on subspaces under which norm retrieval holds. Not only the dimension of each subspace can be important but also as the following propositions suggests, the sum of the dimensions also can be important.

Proposition 2.0.23. If a family of subspaces $\left\{W_{i}\right\}_{i=1}^{n}$ in $\mathbb{R}^{m}$ does norm retrieval then $\sum_{i=1}^{n} \operatorname{dim} W_{i} \geq m$.

Moreover, if $\sum_{i=1}^{n} k_{i}=L m$ and $k_{i}>m$ then there exist $\left\{W_{i}\right\}_{i=1}^{n}$ doing norm retrieval where $\operatorname{dim} W_{i}=k_{i}$ for each $1 \leq i \leq n$.

Proof. If $\sum_{i=1}^{n} \operatorname{dim} W_{i}<m$ then we may pick non-zero $x$ such that $x \perp W_{i}$ for each $i \in[n]$ so that $\left\|P_{i} x\right\|=0$ for all $i \in[n]$ and therefore $\left\{W_{i}\right\}_{i=1}^{n}$ fails norm retrieval. To prove the second part, let $\left\{g_{i}\right\}_{i=1}^{m}$ be an orthonormal basis for $\mathbb{R}^{m}$. We represent this basis $L$-times as a multiset:

$$
\left\{\phi_{i}\right\}_{i=1}^{L m}=:\left\{g_{1}, \ldots, g_{m}, g_{1}, \ldots, g_{m}, \ldots, g_{1}, \ldots, g_{m}\right\}
$$

and index it as: $\left\{e_{i}\right\}_{i=1}^{L m}$. We may pick a partition of $[L m]$ in the following manner:

$$
\begin{aligned}
& I_{1}=\left\{1,2, \ldots, k_{1}\right\} \\
& I_{2}=\left\{k_{1}+1, \ldots, k_{1}+k_{2}\right\} \\
& I_{3}=\left\{k_{1}+k_{2}+1, \ldots, k_{1}+k_{2}+k_{3}\right\}
\end{aligned}
$$

Now define $W_{i}=\operatorname{span}\left\{e_{j}\right\}_{j \in I_{i}}$ with respective projections $P_{i}$. Then if $x=\sum_{j=1}^{m} a_{j} e_{j}$ 
then

$$
\sum_{i=1}^{n}\left\|P_{i} x\right\|^{2}=\sum_{i=1}^{n} \sum_{j \in I_{i}}\left|a_{j}\right|^{2}=L \sum_{j=1}^{m}\left|a_{j}\right|^{2}=L\|x\|^{2}
$$

Hence the result.

Notice that the above proposition may fail if $\sum_{i=1}^{n} k_{i} \neq L m$.

\subsubsection{Phase Retrieval and Norm Retrieval}

It was shown in [3] if a family of projections $\left\{P_{i}\right\}_{i=1}^{n}$ yield phase retrieval then the complements might not yields phase retrieval. The main reason to study norm retrieval is to see how norm retrieval is vital in connecting phase retrieval to the complements. In this section, we will study in detail the relationship between norm retrieval and phase retrieval. We will also give a number of examples to illustrate norm retrieval and phase retrieval.

The following theorem of Edidin [9] is significant in phase retrieval as it gives a necessary and sufficient condition for subspaces to do phase retrieval.

Theorem 2.0.24. [9] A family of projections $\left\{P_{i}\right\}_{i=1}^{n}$ in $\mathbb{R}^{m}$ does phase retrieval if and only if for every $0 \neq x \in \mathbb{R}^{m}$, the vectors $\left\{P_{i} x\right\}_{i=1}^{n}$ span the space.

Corollary 2.0.25. If $\left\{W_{i}\right\}_{i=1}^{n}$ in $\mathbb{H}^{m}$ does phase retrieval, then $\left\{W_{i}^{\perp}\right\}_{i=1}^{n}$ spans the space.

Proof. By the way of contradiction, suppose $\left\{W_{i}{ }^{\perp}\right\}_{i=1}^{n}$ does not span $\mathbb{H}^{m}$. There exists $0 \neq x \perp W_{i}^{\perp}$ for all $i$. Therefore $x \in W_{i}$ for all $i$ and

$$
P_{i} x=x \quad \text { for all } i \in[n]
$$

Therefore, $\left\{P_{i} x\right\}$ can not span $\mathbb{H}^{m}$. Thus ${ }_{26}$ by Theorem 2.0.24, $\left\{W_{i}\right\}_{i=1}^{n}$ does not do 
phase retrieval.

Corollary 2.0.26. Let $\left\{W_{i}\right\}_{i=1}^{n}$ be a collection of subspaces of $\mathbb{R}^{m}$ with $P_{i}$ denoting the projection onto $W_{i}$ for each $i \in[n]$. If $\left\{W_{i}\right\}_{i=1}^{n}$ does phase retrieval in $\mathbb{R}^{m}$ then for every $I \subset[n]$ with $|I| \leq m-2$, the collection $\left\{W_{i}^{\perp}\right\}_{i \in I^{c}}$ spans $\mathbb{R}^{m}$.

Proof. By way of contradiction, suppose $\left\{W_{i}^{\perp}\right\}_{i \in I^{c}}$ dose not span the space, pick a non-zero $x \perp\left\{W_{i}^{\perp}\right\}$ for all $i \in I^{c}$. This implies $x \in \bigcap_{i \in I^{c}} W_{i}$ and therefore $\left\{P_{i} x\right\}_{i=1}^{n}$ contains at most $m-1$ distinct vectors and can not span $\mathbb{R}^{m}$. This contradicts the theorem 2.0.24.

The following example shows that it is possible for subspaces to do norm retrieval even if the complements do not span the space which we see as one of the main differences between phase retrieval and norm retrieval.

Example 2.0.1. Let $\left\{e_{i}\right\}_{i=1}^{3}$ be a orthonormal basis for $\mathbb{R}^{3}$, then let

$$
\begin{array}{ll}
W_{1}=\operatorname{span}\left\{e_{1}, e_{2}\right\} & W_{1}^{\perp}=\operatorname{span}\left\{e_{3}\right\} \\
W_{2}=\operatorname{span}\left\{e_{2}, e_{3}\right\} & W_{2}^{\perp}=\operatorname{span}\left\{e_{1}\right\} \\
W_{3}=\operatorname{span}\left\{e_{2}\right\} & W_{3}^{\perp}=\operatorname{span}\left\{e_{1}, e_{3}\right\}
\end{array}
$$

Observe that:

$$
\|x\|^{2}=\left\|P_{1} x\right\|^{2}+\left\|P_{2} x\right\|^{2}-\left\|P_{3} x\right\|^{2}
$$

so, we can see $\left\{W_{i}\right\}_{i=1}^{3}$ does norm retrieval but $\left\{W_{i}\right\}^{\perp}, i=1,2,3$ do not span $\mathbb{R}^{3}$.

Notice that in general, if $W_{1}=\mathbb{H}^{m}$, then $W_{1}$ itself does norm retrieval while $W_{1}^{\perp}=$ $\{0\}$. 
We realized so far from the definitions that any collection of subspaces which do phase retrieval yields norm retrieval. However, the converse need not hold true always. For instance, any orthonormal basis does norm retrieval in $\mathbb{R}^{m}$ but it has too few vectors to do phase retrieval. Notice that we need at least $2 m-1$ vectors to do phase retrieval in $\mathbb{R}^{m}$.

Given subspaces $\left\{W_{i}\right\}_{i=1}^{n}$ of $\mathbb{H}^{m}$ which yield phase retrieval, it is not necessarily true that $\left\{W_{i}^{\perp}\right\}_{i=1}^{n}$ does phase retrieval. The following result proves that norm retrieval is the condition needed to pass phase retrieval to orthogonal complements. Though the result is already proved in [4], we include it here for completeness.

Lemma 2.0.27. Suppose the family of subspaces $\left\{W_{i}\right\}_{i=1}^{n}$ does phase retrieval and $\left\{P_{i}\right\}_{i=1}^{n}$ are their respective projections. Then $\left\{W_{i}^{\perp}\right\}_{i=1}^{n}$ does phase retrieval if and only if $\left\{W_{i}^{\perp}\right\}_{i=1}^{n}$ does norm retrieval.

Proof. Assume that $\left\|\left(I-P_{i}\right) x\right\|=\left\|\left(I-P_{i}\right) y\right\|$ for all $i \in[n]$ and $\left\{\left(I-P_{i}\right)\right\}_{i=1}^{n}$ does norm retrieval i.e. $\|x\|=\|y\|$. Then

$$
\left\|\left(I-P_{i}\right) x\right\|^{2}=\|x\|^{2}-\left\|P_{i} x\right\|^{2}=\|y\|^{2}-\left\|P_{i} y\right\|^{2}=\left\|\left(I-P_{i}\right) y\right\|^{2} .
$$

Since $\|x\|=\|y\|$, we have

$$
\left\|P_{i} x\right\|=\left\|P_{i} y\right\| \text { for all } i \in[n] .
$$

Since $\left\{P_{i}\right\}_{i=1}^{n}$ does phase retrieval, it follows that $x=c y$ for some $|c|=1$.

The other direction of the theorem is clear.

Next is an example of a family of subspaces $\left\{W_{i}\right\}_{i=1}^{n}$ which does phase retrieval but the complement subspaces fail to do phase retrieval and hence fail norm retrieval [3]. 
Example 2.0.2. Let $\left\{\phi_{i}\right\}_{i=1}^{3}$ and $\left\{\psi_{i}\right\}_{i=1}^{3}$ be orthonormal bases for $\mathbb{R}^{3}$ such that $\left\{\phi_{i}\right\}_{i=1}^{3} \cup\left\{\psi_{i}\right\}_{i=1}^{3}$ is full spark. Consider the subspaces

$$
\begin{array}{ll}
W_{1}=\operatorname{span}\left(\left\{\phi_{1}, \phi_{3}\right\}\right) & W_{1}{ }^{\perp}=\operatorname{span}\left(\left\{\phi_{2}\right\}\right) \\
W_{2}=\operatorname{span}\left(\left\{\phi_{2}, \phi_{3}\right\}\right) & W_{2}{ }^{\perp}=\operatorname{span}\left(\left\{\phi_{1}\right\}\right) \\
W_{3}=\operatorname{span}\left(\left\{\phi_{3}\right\}\right) & W_{3}{ }^{\perp}=\operatorname{span}\left(\left\{\phi_{1}, \phi_{2}\right\}\right) \\
W_{4}=\operatorname{span}\left(\left\{\psi_{1}\right\}\right) & W_{4}{ }^{\perp}=\operatorname{span}\left(\left\{\psi_{2}, \psi_{3}\right\}\right) \\
W_{5}=\operatorname{span}\left(\left\{\psi_{2}\right\}\right) & W_{5}{ }^{\perp}=\operatorname{span}\left(\left\{\psi_{1}, \psi_{3}\right\}\right)
\end{array}
$$

Then $\left\{W_{i}\right\}_{i=1}^{5}$ allows phase retrieval for $\mathbb{R}^{3}$ while the orthogonal complements $\left\{W_{i}^{\perp}\right\}_{i=1}^{5}$ do not.

The following corollary states that if a family of vectors contains an orthonormal basis then phase retrieval can be transferred to the complements.

Corollary 2.0.28. If a family of vectors $\left\{\phi_{i}\right\}_{i=1}^{n}$ does phase retrieval and contains an orthonormal basis, then $\left\{\phi_{i}^{\perp}\right\}_{i=1}^{n}$ does phase retrieval.

Proof. If $\left\{\phi_{i}\right\}_{i \in I}$ is an orthonormal basis for some $I \subseteq[n]$, then $\left\{\phi_{i}^{\perp}\right\}_{i \in I}$ does norm retrieval. Hence so does the larger set $\left\{\phi_{i}^{\perp}\right\}_{i=1}^{n}$. Since $\left\{\phi_{i}\right\}_{i=1}^{n}$ does phase retrieval, and $\left\{\phi_{i}^{\perp}\right\}_{i=1}^{n}$ does norm retrieval, we can conclude the latter does phase retrieval as well which follows from Lemma 2.0.27.

In order to characterize subspaces satisfying norm retrieval, we need to study the conditions under which norm retrieval holds and also the cases when norm retrieval fails. The next result gives us a sufficient condition for the subspaces to do norm retrieval. It is enough to check if the identity is in the linear span of the projections in order for the subspaces to do norm retrieval. A similar result in the case of phase retrieval is proved in [4]. 
Proposition 2.0.29. [4] Let $\left\{W_{i}\right\}_{i=1}^{n}$ be subspaces of $\mathbb{R}^{m}$ with corresponding projections $\left\{P_{i}\right\}_{i=1}^{n}$. Then the projections, $\left\{P_{i}\right\}_{i=1}^{n}$ do norm retrieval, if for all $i \in[n]$, there exist $a_{i} \in \mathbb{R}^{m}$ such that $\sum_{i=1}^{n} a_{i} P_{i}=I$, .

Proof. Given a vector $x \in \mathbb{R}^{m}$, then

$$
\begin{aligned}
\|x\|^{2}=\langle x, x\rangle & =\left\langle\sum_{i=1}^{n} a_{i} P_{i} x, x\right\rangle=\sum_{i=1}^{n} a_{i}\left\langle P_{i} x, x\right\rangle \\
& =\sum_{i=1}^{n} a_{i}\left\langle P_{i} x, P_{i} x\right\rangle=\sum_{i=1}^{n} a_{i}\left\|P_{i} x\right\|^{2} .
\end{aligned}
$$

Since for each $i \in[n]$, the coefficients $a_{i}$ and $\left\|P_{i} x\right\|$ are known, the collection $\left\{P_{i}\right\}_{i=1}^{n}$ does norm retrieval.

A counter example for the converse of the above proposition is given in [4] where the authors construct a collection of projections, $\left\{P_{i}\right\}_{i=1}^{n}$, which does phase retrieval but $\mathrm{I} \notin \operatorname{span}\left\{P_{i}\right\}_{i=1}^{n}$. To show it for the real case, For $2 m \leq n \leq m(m+1) / 2$ choose any full spark frame $\Phi=\left\{\phi_{i}\right\}_{i=1}^{n}$ for $\mathbb{R}^{m}$ such that $\left\{\phi_{i} \phi_{i}^{*}\right\}_{i=1}^{n}$ is linearly independent. Let $S$ be the frame operator for $\Phi$ and define

$$
\psi_{i}=\frac{S^{-1 / 2} \phi_{i}}{\left\|S^{-1 / 2} \phi_{i}\right\|}
$$

with $P_{i}=\psi_{i} \psi_{i}^{*}$. When $P_{i}$ is a rank one orthogonal projection. Since $\left\{S^{-1 / 2} \phi_{i}\right\}_{i=1}^{n}$ is a Parseval frame it follows that

$$
\mathrm{I}=\sum_{i=1}^{n}\left\|S^{-1 / 2} \phi_{i}\right\|^{2} P_{i}
$$

Also, since $\left\{\phi_{i}\right\}_{i=1}^{n}$ is linearly independent it follows that $\left\{P_{i}\right\}_{i=1}^{n}$ is linearly independent and therefore the above equality is the only way to write $I$ as a linear combination of the $\left\{P_{i}\right\}^{\prime} s$. 
Also since $\left\{\phi_{i}\right\}_{i=1}^{n}$ is full spark, we know that $\left\|S^{-1 / 2} \phi_{i}\right\| \neq 0$ for every $i \in[n]$. Therefore it follows that if $\mathcal{I} \subseteq[n]$ then $I \notin \operatorname{span}\left\{P_{i}\right\}_{i \in \mathcal{I}}$. Furthermore, since $n \geq 2 m$ and $\left\{\phi_{i}\right\}_{i=1}^{n}$ is full spark it follows that $\left\{P_{i}\right\}_{i \in \mathcal{I}}$ yields phase retrieval (and hence norm retrieval) whenever $|\mathcal{I}| \geq 2 m-1$.

Here, we provide another example for the same reason. We give a set of five vectors in $\mathbb{R}^{3}$ which does phase retrieval; however the identity operator is not in the span of these vectors. We need the following theorem that provides a necessary and sufficient condition for a frame not to be scalable in $\mathbb{R}^{3}$. Recall that a frame $\left\{\phi_{i}\right\}_{i=1}^{n} \in \mathbb{R}^{m}$ is said to be scalable if there exists scalars $c_{i} \geq 0, i \in[n]$ such that $\left\{c_{i} \phi_{i}\right\}_{i=1}^{n}$ is a Parseval frame [13]. Later in the next section, we prove that scalable frames always do norm retrieval.

Theorem 2.0.30. [13] A frame $\phi$ in $\mathbb{R}^{3}-\{0\}$ for $\mathbb{R}^{3}$ is not scalabale iff all frame vectors of $\phi$ are contained in an interior of an elliptical conical surface with vertex 0 and intersecting the corners of a rotated unit cube.

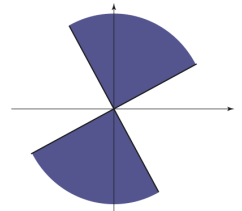

(a)

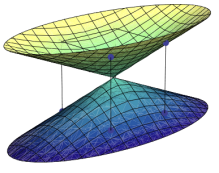

(b)

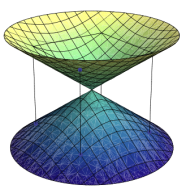

(c)

Figure 2.1: (a) shows a sample region of vectors of a non scalable frame in $\mathbb{R}^{2}$. (b) and (c) show examples of $C_{3}^{-}$and $C_{3}^{+}$which determine sample regions in $\mathbb{R}^{3}$.

The next example is a counter example for the converse of 2.0.29 using a family of five vectors in $\mathbb{R}^{3}$.

Example 2.0.3. A frame $\left\{\phi_{i}\right\}_{i=1}^{5}$ in $\mathbb{R}^{3}$ which does phase retrieval but

$$
\sum_{i=1}^{5} a_{i} \phi_{i} \neq I, \text { for any } a_{i} \in \mathbb{R}
$$


Choose five full spark vectors in the cone referred in the previous theorem 2.0.30. These vectors do phase retrieval and hence norm retrieval in $\mathbb{R}^{3}$.

Now, given $a_{i} \in \mathbb{R}$,

$$
\sum_{i=1}^{5} a_{i} \phi_{i}=\sum_{i=1}^{5}\left|a_{i}\right|\left(\epsilon_{i} \phi_{i}\right) \quad \text { for } \epsilon_{i}= \pm 1
$$

but, $\epsilon_{i} \phi_{i}$ is still inside the cone for each $i$. Therefore $\sum_{i=1}^{5}\left|a_{i}\right|\left(\epsilon_{i} \phi_{i}\right) \neq I$.

We fully studied the cases when subspaces do phase retrieval and norm retrieval. Now we want to study the conditions under which the complement subspaces $\left\{W_{i}\right\}_{i \in \mathcal{I}^{c}}$ satisfy norm retrieval when the family of subspaces $\left\{W_{i}\right\}_{i \in \mathcal{I}}$ do norm retrieval. The next proposition gives a sufficient condition for the complements to satisfy norm retrieval when the subspaces do.

Proposition 2.0.31. If $\left\{W_{i}\right\}_{i=1}^{n}$ are subspaces of $\mathbb{R}^{m}$ with corresponding projections $\left\{P_{i}\right\}_{i=1}^{n}$ such that $\sum_{i=1}^{n} a_{i} P_{i}=I$ and $\sum_{i=1}^{n} a_{i} \neq 1$. Then $\left\{I-P_{i}\right\}_{i=1}^{n}$ does norm retrieval.

Proof. Observe the following

$$
\sum_{i=1}^{n} a_{i}\left(I-P_{i}\right)=\left(\sum_{i=1}^{n} a_{i}\right) I-\sum_{i=1}^{n} a_{i} P=\left(\sum_{i=1}^{n} a_{i}\right) I-I=\left(\sum_{i=1}^{n} a_{i}-1\right) I
$$

Let $\alpha=\sum_{i=1}^{n} a_{i}-1$ then a short calculation shows $\sum_{i=1}^{n} \frac{a_{i}}{\alpha}\left(I-P_{i}\right)=I$. By the previous proposition this shows $\left\{I-P_{i}\right\}_{i=1}^{n}$ does norm retrieval.

It is possible that $\sum a_{i} P_{i}=I=\sum b_{i} P_{i}$ with $\sum a_{i}=1$ but $\sum b_{i} \neq 1$, as we will see in the following example. 
Example 2.0.4. Let $\left\{e_{i}\right\}_{i=1}^{3}$ be an orthonormal basis for $\mathbb{R}^{3}$. Now let

$$
\begin{array}{ll}
W_{1}=\operatorname{span}\left\{e_{1}\right\} & W_{1}{ }^{\perp}=\operatorname{span}\left\{e_{2}, e_{3}\right\} \\
W_{2}=\operatorname{span}\left\{e_{2}\right\} & W_{2}{ }^{\perp}=\operatorname{span}\left\{e_{1}, e_{3}\right\} \\
W_{3}=\operatorname{span}\left\{e_{3}\right\} & W_{3}{ }^{\perp}=\operatorname{span}\left\{e_{1}, e_{2}\right\} \\
W_{4}=\operatorname{span}\left\{e_{1}, e_{2}\right\} & W_{4}{ }^{\perp}=\operatorname{span}\left\{e_{3}\right\} \\
W_{5}=\operatorname{span}\left\{e_{1}, e_{3}\right\} & W_{5}{ }^{\perp}=\operatorname{span}\left\{e_{2}\right\}
\end{array}
$$

Both $\left\{W_{i}\right\}$ and $\left\{W_{i}^{\perp}\right\}$ do norm retrieval. Let $P_{i}$ denote the projections on to $W_{i}$, then

$$
\sum_{i=1}^{5} a_{i} P_{i}=P_{1}+P_{2}+P_{3}+0 \cdot P_{4}+0 \cdot P_{5}=I
$$

and

$$
\sum_{i=1}^{5} b_{i} P_{i}=-P_{1}+0 \cdot P_{2}+0 \cdot P_{3}+P_{4}+P_{5}=I
$$

However, $\sum_{i=1}^{5} a_{i}=3 \neq 1=\sum_{i=1}^{5} b_{i}$.

\subsubsection{Classification of Norm Retrieval}

In this section, we give a detailed classification of norm retrieval by projections. The following theorem in [22] uses the span of the frame elements to classify norm retrievable frames in $\mathbb{R}^{m}$.

Theorem 2.0.32. [22] A frame $\left\{\phi_{i}\right\}_{i=1}^{n} \subset \mathbb{R}^{m}$ does norm retrieval if and only if for any partition $\left\{I_{j}\right\}_{j=1}^{2}$ for $j \in[n]$, span $\left\{\phi_{i}\right\}_{i \in I_{1}}^{\perp} \perp \operatorname{span}\left\{\phi_{i}\right\}_{i \in I_{2}}^{\perp}$.

Next, we prove one of the main results of this paper. This is an extension of the previous Theorem 2.0.32 and it fully classifies the subspaces of $\mathbb{R}^{m}$ which do norm retrieval. 
Theorem 2.0.33. Let $\left\{P_{i}\right\}_{i=1}^{n}$ be projections onto subspaces $\left\{W_{i}\right\}_{i=1}^{n}$ of $\mathbb{R}^{m}$. Then the following are equivalent:

1. $\left\{P_{i}\right\}_{i=1}^{n}$ does norm retrieval,

2. Given any orthonormal bases $\left\{\phi_{i j}\right\}_{j=1}^{I_{i}}$ of $W_{i}$ and any subcollection $S \subseteq\{(i, j)$ : $\left.1 \leq i \leq n, 1 \leq j \leq I_{i}\right\}$ then

$$
\operatorname{span}\left\{\phi_{i j}\right\}_{(i, j) \in S}^{\perp} \perp \operatorname{span}\left\{\phi_{i j}\right\}_{(i, j) \in S^{c}}^{\perp},
$$

3. For any orthonormal basis $\left\{\phi_{i j}\right\}_{j=1}^{I_{i}}$ of $W_{i}$, then the collection of vectors $\left\{\phi_{i j}\right\}_{i=1, j \in I_{i}}^{n}$ do norm retrieval.

Proof. $(1) \Rightarrow(2):$ Suppose $x \in \operatorname{span}\left\{\phi_{i j}\right\}_{(i, j) \in S}^{\perp}$, and $y \in \operatorname{span}\left\{\phi_{i j}\right\}_{(i, j) \in S^{c}}^{\perp}$ and let $I=[n]$ then,

$$
\begin{aligned}
\left\|P_{i}(x+y)\right\|^{2} & =\sum_{j=1}^{I_{i}}\left|\left\langle x+y, \phi_{i j}\right\rangle\right|^{2} \\
& =\sum_{j \in I \cap I_{i}}\left|\left\langle x, \phi_{i}\right\rangle\right|^{2}+\sum_{j \in I^{c} \cap I_{i}}\left|\left\langle y, \phi_{i}\right\rangle\right|^{2} \\
& =\sum_{j=1}^{I_{i}}\left|\left\langle x-y, \phi_{i j}\right\rangle\right|^{2} \\
& =\left\|P_{i}(x-y)\right\|^{2}
\end{aligned}
$$

Since $\left\{P_{i}\right\}_{i=1}^{n}$ does norm retrieval, we have

$$
\|x+y\|^{2}=\|x\|^{2}+\|y\|^{2}+2\langle x, y\rangle=\|x-y\|^{2}=\|x\|^{2}+\|y\|^{2}-2\langle x, y\rangle,
$$

and so $\langle x, y\rangle=0$ 
$(2) \Rightarrow(1)$ : Assume that $\left\|P_{i} x\right\|=\left\|P_{i} y\right\|$ for all $1 \leq i \leq n$. Then, by $([3])$ we can find an orthonormal basis $\left(\phi_{i j}\right)_{j=1}^{K_{i}}$ for $W_{i}$ such that

$$
\left|\left\langle\phi_{i j}, x\right\rangle\right|=\left|\left\langle\phi_{i j}, y\right\rangle\right| \quad \forall i, j
$$

Denote $A=\left\{(i, j):\left\langle\phi_{i j}, x\right\rangle=\left\langle\phi_{i j}, y\right\rangle\right\}$ and $B=\left\{(i, j):\left\langle\phi_{i j}, x\right\rangle=-\left\langle\phi_{i j}, y\right\rangle\right\}$. Now we can see that

$$
(x-y) \perp \operatorname{span}\left\{\phi_{i j}:(i, j) \in A\right\}
$$

and also

$$
(x+y) \perp \operatorname{span}\left\{\phi_{i j}:(i, j) \in B\right\}
$$

By (2), we must have that $\langle x+y, x-y\rangle=\|x\|^{2}-\|y\|^{2}=0$, which implies that $x$ and $y$ have the same norm.

The third equivalence is immediate from the result in Theorem (2.0.32).

The next Corollary will show that all scalable frames satisfy norm retrieval.

Corollary 2.0.34. If $\Phi=\left\{\phi_{i}\right\}_{i=1}^{n}$ does norm retrieval then $\Phi^{\prime}=\left\{c_{i} \phi_{i}\right\}_{i=1}^{n}, c_{i} \neq 0$ does norm retrieval. Hence all scalable frames do norm retrieval.

Proof. This is an immediate result of Theorem 2.0.33. Notice that the conditions in Theorem 2.0.33 do not depend on the norm of each vector $\phi_{i}$.

For the complex case we have:

Proposition 2.0.35. If $\left\{P_{i}\right\}_{i=1}^{n}$ does norm retrieval, then if we choose orthonormal bases $\left\{\phi_{i, j}\right\}_{j=1}^{I_{i}}$ of $W_{i}$ and any subcollection $S \subseteq\left\{(i, j): 1 \leq i \leq n, 1 \leq j \leq I_{i}\right\}$ then

$$
x \perp \operatorname{span}\left\{\phi_{i j}\right\}_{(i, j) \in S} \text { and } y \perp \operatorname{span}\left\{\phi_{i j}\right\}_{(i, j) \in S^{c}}
$$


implies:

$$
\operatorname{Re}\langle x, y\rangle=0
$$

Proof. Given $x, y$ as above:

$$
\left|\left\langle x+y, \phi_{i j}\right\rangle\right|=\left|\left\langle x-y, \phi_{i j}\right\rangle\right| \quad \text { for all }(i, j)
$$

Since our vectors do norm retrieval, we have

$$
\|x+y\|^{2}=\|x\|^{2}+\|y\|^{2}+2 \operatorname{Re}\langle x, y\rangle=\|x-y\|^{2}=\|x\|^{2}+\|y\|^{2}-2 \operatorname{Re}\langle x, y\rangle,
$$

and so $\operatorname{Re}\langle x, y\rangle=0$.

One of the interesting results about norm retrieval is that we can show if a set of $n$-vectors in $\mathbb{R}^{m}$ satisfy norm retrieval then they are orthogonal which is not necessarily true for phase retrieval. Theorem 2.0.33 was helpful in simplifying the proof of such a theorem which was stated in [3]. The following is a simple proof of a result in [3] which has a very complicated proof in that paper.

Corollary 2.0.36. If $\left\{\phi_{i}\right\}_{i=1}^{m}$ do norm retrieval in $\mathbb{R}^{m}$, then the vectors are orthogonal.

Proof. Suppose we have a set of vectors $\left\{\phi_{i}\right\}_{i=1}^{n}$ such that $\left\|\phi_{i}\right\|=1$ and that for some $j \in[n], \phi_{j}$ is not orthogonal to $\operatorname{span}\left\{\phi_{i}\right\}_{i \neq j}$. Now, choose a unit vector $x$ such that $x \perp \phi_{i}$ for all $i \neq j$. Let vector $y$ be such that

$$
y=x-\left\langle x, \phi_{j}\right\rangle \phi_{j}
$$

now,

$$
\left\langle\phi_{j}, y\right\rangle=\left\langle\phi_{j}, x\right\rangle-{ }_{36}\left\langle x, \phi_{j}\right\rangle\left\langle\phi_{j}, \phi_{j}\right\rangle=0
$$


Let $I=\{i: i \neq j\}$, then

$$
x \perp \operatorname{span}\left\{\phi_{i}\right\}_{i \in I} \text { and } y \perp \phi_{j},
$$

but

$$
\langle x, y\rangle=\langle x, x\rangle-\left\langle x, \phi_{j}\right\rangle\left\langle x, \phi_{j}\right\rangle=1-\left|\left\langle x, \phi_{j}\right\rangle\right|^{2} \neq 0,
$$

contradicting the theorem.

In order to classify norm retrieval, we will need to find the correlation between the subspaces and the complement subspaces and study the conditions under which norm retrieval is possible.

Corollary 2.0.37. Consider a frame $\Phi=\left\{\phi_{i}\right\}_{i=1}^{n}$. The followings are equivalent:

1. $\Phi$ does norm retrieval.

2. For $i \in[n]$ if $W_{1}=\operatorname{span}\left\{\phi_{i}\right\}_{i \in I}$ and $W_{2}=\operatorname{span}\left\{\phi_{i}\right\}_{i \in I^{c}}$ then, $W_{1}{ }^{\perp} \subseteq W_{2}$.

Proof. By Theorem 2.0.33, it follows that:

$$
\begin{aligned}
\Phi \text { does norm retrieval } & \Longleftrightarrow W_{1}{ }^{\perp} \perp W_{2}{ }^{\perp} \\
& \Longleftrightarrow W_{1}{ }^{\perp} \subseteq W_{2}
\end{aligned}
$$

and this proves the theorem.

Both phase retrieval and norm retrieval are preserved when applying projections to the vectors. Also, if we apply any invertible operator, phase retrieval will be preserved. To learn more about the details we refer to [4]. On the other hand, this is not the case with norm retrieval, in general. 
The next corollary will show that applying operators will not keep the norm retrieval properties. Previously we discussed that it is possible to use invertible operators to lift the properties of phase retrieval but we will see that is not the case for norm retrieval.

Corollary 2.0.38. Norm retrieval is not preserved under the application of an invertible operator, in general.

Proof. Let $\phi=\left\{\phi_{i}\right\}_{i=1}^{m}$ be linearly independent vectors in $\mathbb{R}^{m}$ which are not orthogonal. Since all the vectors satisfying norm retrieval are orthogonal (Corollary 2.0.36), $\Phi$ cannot do norm retrieval. But there exists an invertible operator $T$ on $\mathbb{R}^{m}$ so that $\left\{T \phi_{i}\right\}_{i=1}^{m}$ is an orthonormal basis and so does norm retrieval.

However, notice that unitary operators, which are invertible, do preserve norm retrieval.

One of the classifications of norm retrieval with respect to the Parseval frames suggests that if we partition the frame into two sets, then the vectors chosen from each set would be orthogonal to each other. The following corollary holds in the infinite dimensional case. The proof is the same as the previous corollary.

Corollary 2.0.39. If $\Phi$ is a Parseval frame, it does norm retrieval. Hence, if we partition $\Phi$ into two disjoint sets, and choose a vector orthogonal to each set, then these vectors are orthogonal.

Proof. Let $\Phi=\left\{\phi_{i}\right\}_{i \in I}$ be a Parseval frame and $J \subseteq I$. Let $T$ be its analysis operator. If

$$
x \perp\left\{\phi_{i}\right\}_{i \in J} \text { and } y \perp\left\{\phi_{i}\right\}_{i \in J^{c}}
$$

then $T x=\left(\left\langle x, \phi_{i}\right\rangle\right)$ and $T y=\left(\left\langle y, \phi_{i}\right\rangle\right)$ do not have any nonzero coordinates in common. Therefore, 


$$
T x \perp T y
$$

Since the analysis operator of a Parseval frame is an isometry, we have $x \perp y$.

We can generalize the following classical result in frame theory in Parseval frames:

Property 2.0.40. Suppose $\left\{\phi_{i}\right\}_{i \in I}$ is a Parseval frame. Let the subspace $W$ be such that $W=\operatorname{span}\left\{\phi_{i}\right\}_{i \neq j}$. Now, if $\phi_{j} \notin W$ then $\phi_{j} \perp W$.

It turns out that a much more general result holds.

Corollary 2.0.41. Let $\left\{\phi_{i}\right\}_{i=1}^{n}$ be a Parseval frame in $\mathbb{R}^{m}$. For $I \subseteq[n]$, let $W_{I}=$ $\operatorname{span}\left\{\phi_{i}\right\}_{i \in I}$ and $W_{I^{c}}=\operatorname{span}\left\{\phi_{i}\right\}_{i \in I^{c}}$. If $W_{I} \bigcap W_{I^{c}}=\{0\}$, then $W_{I} \perp W_{I^{c}}$.

Corollary 2.0.42. Suppose that a frame $\Phi=\left\{\phi_{i}\right\}_{i=1}^{n}$ in $\mathbb{R}^{m}$ satisfies norm retrieval. Let $S$ be the frame operator, then for every $I \subset[m]$, if $x \perp \operatorname{span}\left\{\phi_{i}\right\}_{i \in I}$ then $x \in$ $\operatorname{span}\left\{S^{-1} \phi_{i}\right\}_{i \in I^{c}}$

In particular, if $\Phi$ is a Parseval frame, then $x \in \operatorname{span}\left\{\phi_{i}\right\}_{i \in I^{c}}$.

Proof. Given $x$ such that $x \perp \operatorname{span}\left\{\phi_{i}\right\}_{i \in I}$,

$$
\begin{aligned}
x & =\sum_{i=1}^{n}\left\langle x, \phi_{i}\right\rangle S^{-1} \phi_{i} \\
& =\sum_{i \in I^{c}}\left\langle x, \phi_{i}\right\rangle S^{-1} \phi_{i}
\end{aligned}
$$

We next provide a classification of norm retrieval using Naimark's theorem. It turns out that every frame can be scaled to look similar to Naimark's theorem. 
Proposition 2.0.43. If $\left\{\phi_{i}\right\}_{i=1}^{n}$ is a frame with Bessel bound 1 in $\mathbb{R}^{m}$, then there is an isometry $T: \mathbb{R}^{m} \rightarrow \ell_{2}^{2 n-1}$ with orthonormal basis $\left\{e_{i}\right\}_{i=1}^{2 n-1}$ so that the orthogonal projection onto $\mathbb{R}^{m}$ satisfies:

$$
P e_{i}=T \phi_{i} \quad \text { for every } i \in[n]
$$

Proof. Let $\left\{g_{i}\right\}_{i=1}^{m}$ be the eigenbasis for the frame with respective eigenvalues $1=$ $\lambda_{1} \geq \lambda_{2} \geq \cdots \geq \lambda_{m}$. For $n+1 \leq n+i \leq 2 n-1$ let

$$
\phi_{n+i}=\sqrt{1-\lambda_{i+1}} g_{i+1}
$$

Now, for any $\phi \in \mathbb{R}^{m}$ we have

$$
\begin{aligned}
\sum_{i=1}^{2 n-1}\left|\left\langle\phi, \phi_{i}\right\rangle\right|^{2} & =\sum_{i=1}^{n}\left|\left\langle\phi, \phi_{i}\right\rangle\right|^{2}+\sum_{i=n+1}^{2 n-1}\left|\left\langle\phi, \phi_{i}\right\rangle\right|^{2} \\
& =\sum_{i=1}^{n}\left|\lambda_{i}\left\langle\phi, g_{i}\right\rangle\right|^{2}+\sum_{i=1}^{n-1}\left|\left\langle\phi, \sqrt{1-\lambda_{i+1}} g_{i+1}\right\rangle\right|^{2} \\
& =\sum_{i=1}^{n} \lambda_{i}\left|\left\langle\phi, g_{i}\right\rangle\right|^{2}+\sum_{i=2}^{n}\left(1-\lambda_{i}\right)\left|\left\langle\phi, g_{i}\right\rangle\right|^{2} \\
& =\left|\left\langle\phi, g_{1}\right\rangle\right|^{2}+\sum_{i=2}^{n}\left|\left\langle\phi, g_{i}\right\rangle\right|^{2} \\
& =\|\phi\|^{2} .
\end{aligned}
$$

So $\left\{\phi_{i}\right\}_{i=1}^{2 n-1}$ is a Parseval frame. The analysis operator of this Parseval frame $T$ : $\mathbb{R}^{m} \rightarrow \ell_{2}^{2 n-1}$ is then an isometry where

$$
T \phi=\sum_{i=1}^{2 n-1}\left\langle\phi, \phi_{i}\right\rangle e_{i}
$$

where $\left\{e_{i}\right\}_{i=1}^{2 n-1}$ is the unit vector basis of $\ell_{2}^{2 n-1}$. Let $P$ be the orthogonal projection 
of $\ell_{2}^{2 n-1}$ onto $T\left(\mathbb{R}^{m}\right)$. Then given $i \in[2 n-1]$, we have for all $j \in[2 n-1]$ :

$$
\left\langle P e_{i}, T \phi_{j}\right\rangle=\left\langle e_{i}, T \phi_{j}\right\rangle=\left\langle T^{*} e_{i}, \phi_{j}\right\rangle=\left\langle\phi_{i}, \phi_{j}\right\rangle=\left\langle T \phi_{i}, T \phi_{j}\right\rangle
$$

It follows that $P e_{i}=T \phi_{i}$ for all $i \in[n]$.

We can now prove one of the main results in this section.

Theorem 2.0.44. Let $\Phi=\left\{\phi_{i}\right\}_{i=1}^{n}$ be a frame for $\mathbb{R}^{m}$. The following are equivalent:

1. $\Phi$ does norm retrieval.

2. By Proposition 2.0.43 if $T: \mathbb{R}^{m} \rightarrow \ell_{2}^{2 n-1}$ is an isometry and $\left\{e_{i}\right\}_{i=1}^{2 n-1}$ is the unit vector basis for $\ell_{2}^{2 n-1}$ then for every $\phi, \psi \in \mathbb{R}^{m}$ with $\left|\left\langle\phi, \phi_{i}\right\rangle\right|=\left|\left\langle\psi, \phi_{i}\right\rangle\right|$ for all $i \in[n]$, we have

$$
\left\|\sum_{i=n+1}^{2 n-1}\left\langle T \phi, e_{i}\right\rangle e_{i}\right\|^{2}=\sum_{i=n+1}^{2 n-1}\left|\left\langle T \phi, e_{i}\right\rangle\right|^{2}=\left\|\sum_{i=n+1}^{2 n-1}\left\langle T \psi, \phi_{i}\right\rangle e_{i}\right\|^{2}=\sum_{i=n+1}^{2 n-1}\left|\left\langle T \psi, \phi_{i}\right\rangle\right|^{2}
$$

Proof. $(1) \Rightarrow(2)$ : We have for $i \in[n]$,

$$
\left|\left\langle\phi, \phi_{i}\right\rangle\right|=\left|\left\langle T \phi, T \phi_{i}\right\rangle\right|=\left|\left\langle T \phi, P e_{i}\right\rangle\right|=\left|\left\langle T \phi, e_{i}\right\rangle\right|=\left|\left\langle T \psi, T \phi_{i}\right\rangle\right|
$$

By (1), we know that $\|\phi\|=\|T \phi\|=\|\psi\|=\|T \psi\|$. Hence,

$$
\begin{aligned}
\|T \phi\|^{2} & =\sum_{i=1}^{n}\left|\left\langle T \phi_{i}, e_{i}\right\rangle\right|^{2}+\sum_{i=n+1}^{2 n-1}\left|\left\langle T \phi, e_{i}\right\rangle\right|^{2} \\
& =\|\psi\|^{2}=\sum_{i=1}^{n}\left|\left\langle T \phi_{i}, e_{i}\right\rangle\right|^{2}+\sum_{i=n+1}^{2 n-1}\left|\left\langle T \phi, e_{i}\right\rangle\right|^{2}
\end{aligned}
$$


Since $\sum_{i=1}^{n}\left|\left\langle T \phi, e_{i}\right\rangle\right|^{2}=\sum_{i=1}^{n}\left|\left\langle T \psi, e_{i}\right\rangle\right|^{2}$, the result follows.

$(2) \Rightarrow(1)$ : If $\left|\left\langle\phi, \phi_{i}\right\rangle\right|=\left|\left\langle\psi, \phi_{i}\right\rangle\right|$ for all $i \in[n]$ then applying (2) as above we have:

$$
\begin{aligned}
\|\phi\|^{2} & =\|T \phi\|^{2} \\
& =\sum_{i=1}^{n}\left|\left\langle T \phi_{i}, e_{i}\right\rangle\right|^{2}+\sum_{i=n+1}^{2 n-1}\left|\left\langle T \phi, e_{i}\right\rangle\right|^{2} \\
& =\sum_{i=1}^{n}\left|\left\langle T \psi, e_{i}\right\rangle\right|^{2}+\sum_{i=n+1}^{2 n-1}\left|\left\langle T \phi, e_{i}\right\rangle\right|^{2}=\|T \psi\|^{2}
\end{aligned}
$$

so $\|\phi\|=\|\psi\|$ and $\Phi$ does norm retrieval. 


\section{Chapter 3}

\section{Weak Phase Retrieval}

\subsection{Defining weak phase retrieval}

While investigating the relationship between phase retrieval and phaseless reconstruction, in [15] it was noted that if two vectors have the same phase then they will be zero in the same coordinates. This gave way to a weakening of phase retrieval, known as weak phase retrieval. In this work, we study the weakened notions of phase retrieval and phaseless reconstruction. One limitation of current methods used for retrieving the phase of a signal is computing power. Recall that a generic family of $(2 m-1)$ vectors in $\mathbb{R}^{m}$ satisfies phaseless reconstruction, however no set of $(2 m-2)$-vectors can (See [7] for details). By generic we are referring to an open dense set in the set of $(2 m-1)$-element frames in $\mathbb{H}^{m}$. We started with the motivation that weak phase retrieval could be done with $m+1$ vectors in $\mathbb{R}^{m}$. However, it will be shown that the cardinality condition can only be relaxed to $2 m-2$. Nevertheless, the results we obtain in this work are interesting in their own right and contribute to the overall understanding of phase retrieval. We provide illustrative examples in the real and complex cases for weak phase retrieval. 
We start by defining the notion of weak phase retrieval and obtain the minimum number of vectors required to satisfy weak phase retrieval.

Definition 3.1.1. Two vectors in $\mathbb{H}^{m}, x=\left(a_{1}, a_{2}, \ldots, a_{m}\right)$ and $y=\left(b_{1}, b_{2}, \ldots, b_{m}\right)$ weakly have the same phase if there is a $|\theta|=1$ so that

$$
\text { phase }\left(a_{i}\right)=\theta \text { phase }\left(b_{i}\right) \text {, for all } i \in[m] \text { for which } a_{i} \neq 0 \neq b_{i} \text {. }
$$

In the real case, if $\theta=1$ we say $x, y$ weakly have the same signs and if $\theta=-1$ they weakly have opposite signs.

In the definition above note that we are only comparing the phase of $x$ and $y$ for entries where both are nonzero. Hence, two vectors may weakly have the same phase but not have the same phase in the usual sense. We define weak phase retrieval formally as follows:

Definition 3.1.2. A family of vectors $\left\{\phi_{i}\right\}_{i=1}^{n}$ in $\mathbb{H}^{m}$ does weak phase retrieval if for any $x=\left(a_{1}, a_{2}, \ldots, a_{m}\right)$ and $y=\left(b_{1}, b_{2}, \ldots, b_{m}\right)$ in $\mathbb{H}^{m}$, with

$$
\left|\left\langle x, \phi_{i}\right\rangle\right|=\left|\left\langle y, \phi_{i}\right\rangle\right|, \text { for all } i \in[m]
$$

then $x, y$ weakly have the same phase.

Observe that the difference between phase retrieval and weak phase retrieval is that in the later it is possible for $a_{i}=0$ but $b_{i} \neq 0$.

\subsubsection{Real Case}

Now we begin our study of weak phase retrieval in $\mathbb{R}^{m}$. The following proposition provides a useful criteria for determining when two vectors have weakly the same or opposite phases. 
Proposition 3.1.3. Let $x=\left(a_{1}, a_{2}, \ldots, a_{m}\right)$ and $y=\left(b_{1}, b_{2}, \ldots, b_{m}\right)$ in $\mathbb{R}^{m}$. The following are equivalent:

1. We have

$$
\operatorname{sgn}\left(a_{i} a_{j}\right)=\operatorname{sgn}\left(b_{i} b_{j}\right), \quad \text { for all } a_{i} a_{j} \neq 0 \neq b_{i} b_{j} \text {. }
$$

2. Either $x, y$ have weakly the same signs or they have weakly opposite signs.

Proof. $(1) \Rightarrow(2)$ : Let

$$
I=\left\{1 \leq i \leq m: a_{i}=0\right\} \text { and } J=\left\{1 \leq i \leq n: b_{i}=0\right\}
$$

Let

$$
K=[m] \backslash(I \cup J)
$$

So $i \in K$ if and only if $a_{i} \neq 0 \neq b_{i}$. Let $i_{0}=\min K$. We examine two cases:

Case 1: $\operatorname{sgn} a_{i_{0}}=\operatorname{sgn} b_{i_{0}}$.

For any $i_{0} \neq k \in K$, sgn $\left(a_{i_{0}} a_{k}\right)=\operatorname{sgn}\left(b_{i_{0}} b_{k}\right)$, implies sgn $a_{k}=\operatorname{sgn} b_{k}$. Since all other coordinates of either $x$ or $y$ are zero, it follows that $x, y$ weakly have the same signs.

Case 2: $\operatorname{sgn} a_{i_{0}}=-\operatorname{sgn} b_{i_{0}}$.

Is similar the previous case.

$(2) \Rightarrow(1)$ : This is immediate.

The next lemma will be useful in the following proofs as it gives a criteria for showing when vectors do not weakly have the same phase. 
Lemma 3.1.4. Let $x=\left(a_{1}, a_{2}, \ldots, a_{m}\right)$ and $y=\left(b_{1}, b_{2}, \ldots, b_{m}\right)$ be vectors in $\mathbb{R}^{m}$. If there exists $i \in[m]$ such that $a_{i} b_{i} \neq 0$ and $\langle x, y\rangle=0$, then $x$ and $y$ do not have weakly the same or opposite signs.

Proof. We proceed by way of contradiction. If $x$ and $y$ weakly have the same phase then $a_{j} b_{j} \geq 0$ for all $j \in[m]$ and in particular we arrive at the following contradiction

$$
\langle x, y\rangle=\sum_{j=1}^{n} a_{j} b_{j} \geq a_{i} b_{i}>0
$$

If $x$ and $y$ weakly have opposite phases then $a_{j} b_{j} \leq 0$ for all $j \in[m]$ and by reversing the inequalities in the expression above we get the desired result.

In the previous section we talked about the relationship between phase retrieval, phaseless reconstruction and norm retrieval. Now the following result relates weak phase retrieval and phase retrieval. Recall that in the real case, it is known that phase retrieval, phaseless reconstruction and the complement property are equivalent $[7,15]$.

Corollary 3.1.5. Suppose $\Phi=\left\{\phi_{i}\right\}_{i=1}^{n} \in \mathbb{R}^{m}$ does weak phase retrieval but fails complement property, then there exists two vectors $v, w \in \mathbb{R}^{m}$ such that $v \perp w$ and

$$
\left|\left\langle v, \phi_{i}\right\rangle\right|=\left|\left\langle w, \phi_{i}\right\rangle\right| \text { for all } i \in[n]
$$

Further, $v$ and $w$ are disjointly supported.

Proof. By the assumption, $\Phi=\left\{\phi_{i}\right\}_{i=1}^{n}$ fails complement property so there exists $I \subset[n]$, such that $A=\operatorname{span}\left\{\phi_{i}\right\}_{i \in I} \neq \mathbb{R}^{m}$ and $B=\operatorname{span}\left\{\phi_{i}\right\}_{i \in I^{c}} \neq \mathbb{R}^{m}$. Choose $\|x\|=\|y\|=1$ such that $x \perp A$ and $y \perp B$. Then for all $i \in[n]$

$$
\left|\left\langle x+y, \phi_{i}\right\rangle\right|=\left|\left\langle x-y, \phi_{i}\right\rangle\right|
$$


Let $w=x+y$ and $v=x-y$. Then $u \perp v$. Observe

$$
\langle w, v\rangle=\langle x+y, x-y\rangle=\|x\|^{2}+\langle y, x\rangle-\langle x, y\rangle-\|y\|^{2}=0 .
$$

Moreover, the assumption that $\Phi$ does weak phase retrieval implies $u$ and $w$ have weakly the same or opposite phases. Then it follows from Lemma 3.1.4 that $u$ and $w$ are disjointly supported.

Notice that in phase retrieval we proved that in the real case, a set of frame satisfies phase retrieval if and only of it has the complement property. The next example will show that this is not necessarily true for weak phase retrieval. In fact the set of vectors fails complement property but it satisfies weak phase retrieval.

Example 3.1.1. In $\mathbb{R}^{2}$ let $\phi_{1}=(1,1)$ and $\phi_{2}=(1,-1)$. These vectors clearly fail complement property. But if $x=\left(a_{1}, a_{2}\right), b=\left(b_{1}, b_{2}\right)$ and we have,

$$
\left|\left\langle x, \phi_{i}\right\rangle\right|=\left|\left\langle y, \phi_{i}\right\rangle\right|, \text { for } i=1,2,
$$

then

$$
\left|a_{1}+a_{2}\right|^{2}=\left|b_{1}+b_{2}\right|^{2} \text { and }\left|a_{1}-a_{2}\right|^{2}=\left|b_{1}-b_{2}\right|^{2}
$$

By squaring these out and subtracting the result we get:

$$
2 a_{1} a_{2}=2 b_{1} b_{2}
$$

Hence, either $x, y$ have the same signs or opposite signs i.e. These vectors do weak phase retrieval.

The question we can ask here is that under what conditions, a set of vectors would satisfy weak phase retrieval but not phase retrieval. 
With some particular assumptions, the following proposition gives the specific form of vectors which do weak phase retrieval but not phase retrieval.

Proposition 3.1.6. Let $\Phi=\left\{\phi_{i}\right\}_{i=1}^{n}$ be a family of vector in $\mathbb{R}^{m}$ such that $\Phi$ does weak phase retrieval but fails the complement property. Let $x=\left(a_{1}, a_{2}, \ldots, a_{m}\right)$, $y=\left(b_{1}, b_{2}, \ldots b_{m}\right) \in \mathbb{R}^{m}$ such that $x+y \perp x-y$ and satisfy equation 3.1. If $a_{i} b_{i} \neq 0$, $a_{j} b_{j} \neq 0$ for some $i, j$ and all other co-ordinates of $x$ and $y$ are zero, then

$$
\left|a_{i}\right|=\left|b_{i}\right|, \quad \text { for } i=1,2
$$

Proof. Without loss of generality, take $x=\left(a_{1}, a_{2}, 0, \ldots, 0\right)$ and $y=\left(b_{1}, b_{2}, 0, \ldots, 0\right)$. Observe that both $x+y$ and $x-y$ either weakly have the same phase or weakly have the opposite phase. Thus, by Lemma 3.1.4, $x+y$ and $x-y$ have disjoint support as these vectors are orthogonal. Since,

$$
x+y=\left(a_{1}, b_{1}, a_{2}+b_{2}, 0, \ldots, 0\right) \text { and } x-y=\left(a_{1}-b_{1}, a_{2}-b_{2}, 0, \ldots, 0\right)
$$

it reduces to the cases where either $a_{1}=b_{1}, a_{2}=-b_{2}$ or $a_{1}=-b_{1}, a_{2}=b_{2}$. In both cases, it follows from equation 3.1 that $\left|a_{i}\right|=\left|b_{i}\right|$ for all $i \in[m]$.

We have learned that phase retrieval requires at least $2 m-1$ vectors in $\mathbb{R}^{m}$, i.e. a family of vectors $\left\{\phi_{i}\right\}_{i=1}^{n}$ does phase retrieval then $n \geq 2 m-1$. But for weak phase retrieval we were able to show we can get a better bound.

The next theorem gives the main result about the minimum number of vectors required to do weak phase retrieval in $\mathbb{R}^{m}$.

Theorem 3.1.7. If $\left\{\phi_{i}\right\}_{i=1}^{n}$ does weak phase retrieval on $\mathbb{R}^{m}$ then $n \geq 2 m-2$. 
Proof. We proceed by way of contradiction.

Assume $n \leq 2 m-3$. If $I=[m-2]$ then $|I|=m-2$ and $\left|I^{c}\right| \leq m-1$. For this partition of $[n]$, let $x+y$ and $x-y$ be as in Corollary 3.1.5. Then $x+y$ and $x-y$ must be disjointly supported and therefore for each $i \in[m], a_{i}=\epsilon_{i} b_{i}$, where $\epsilon_{i}= \pm 1$. Observe the conclusion holds for a fixed $x$ and any $y \in\left(\operatorname{span}\{\phi\}_{i \in I}\right)^{\perp}$ when $\operatorname{dim}\left(\operatorname{span}\left\{\phi_{i}\right\}_{i \in I}\right)^{\perp} \geq 2$. However this poses a contradiction since there are infinitely many distinct choices of $y$ in this space, while our argument shows that there are at most $2^{m}$ choices of $y$.

Contrary to the initial hopes, the previous result shows that the minimal number of vectors doing weak phase retrieval is only one less than the number of vectors doing phase retrieval. However it is interesting to note that a minimal set of vectors doing weak phase retrieval is necessarily full spark, as is true for the minimal number of vectors doing phase retrieval, as the next result shows.

Theorem 3.1.8. If $\Phi=\left\{\phi_{i}\right\}_{i=1}^{2 m-2}$ does weak phase retrieval in $\mathbb{R}^{m}$, then $\Phi$ is full spark.

Proof. We proceed by way of contradiction. Assume $\Phi$ is not full spark. Then there exists $I \subset\{1,2, \ldots, 2 m-2\}$ with $|I|=m$ such that

$$
\operatorname{dim} \operatorname{span}\left\{\phi_{i}\right\}_{i \in I} \leq m-1
$$

Observe that the choice of $I$ above implies $\left|I^{c}\right|=m-2$. Now we arrive at a contradiction by applying the same argument used in (the proof of) Theorem 3.1.7.

It is important to note that the converse of Theorem 3.1.8 does not hold. In other words, if $\Phi$ is full spark, it does not necessarily do weak phase retrieval. For 
example, the canonical basis in $\mathbb{R}^{2}$ is trivially full spark but does not do weak phase retrieval.

If $\Phi$ is as in Theorem 3.1.8, then the following corollary guarantees it is possible to add a vector to this set and obtain a collection which does phaseless reconstruction and similarly phase retrieval.

Corollary 3.1.9. If $\Phi=\left\{\phi_{i}\right\}_{i=1}^{2 m-2}$ does weak phase retrieval in $\mathbb{R}^{m}$, then there exists a dense set of vectors $\mathrm{F}$ in $\mathbb{R}^{m}$ such that $\{\psi\} \cup \Phi$ does phaseless reconstruction for any $\psi \in \mathrm{F}$.

Proof. The result follows almost instantly from the observation that the set of $\psi \in \mathbb{R}^{m}$ such that $\Phi \bigcup\{\psi\}$ is full spark, it is in fact dense in $\mathbb{R}^{m}$.

To see this let

$$
\mathrm{G}=\bigcup_{\substack{I \subset[2 m-2] \\|I|=m-1}} \operatorname{span}\left\{\phi_{i}\right\}_{i \in I}
$$

Then $\mathrm{G}$ is the finite union of hyperplanes so $\mathrm{G}^{c}$ is dense and $\{\psi\} \bigcup \Phi$ is full spark for any $\psi \in \mathrm{G}^{c}$. It suffices to verify that this collection of vectors is full spark. Either a sub-collection of m-vectors is contained in $\Phi$, then it spans $\mathbb{R}^{m}$, or the subcollection contains the vector $\psi$. In this case, denote $I \subset[2 m-2]$ with $|I|=m-1$ and suppose $\sum_{i \in I} a_{i} \phi_{i}+a \psi=0$. Therefore $a \psi=-\sum_{i \in I} a_{i} \phi_{i}$ and if $a \neq 0$ then:

$$
a \psi \in \operatorname{span}\left\{\phi_{i}\right\}_{i \in I}
$$

which is a contradiction. It follows $a=0$ and since $\Phi$ is full spark (see Theorem 3.1.8), in particular $\left\{\phi_{i}\right\}_{i \in I}$ are linearly independent, it follows that $a_{i}=0$ for all $i \in I$. 


\subsubsection{Complex Case}

An extension of Proposition 3.1.3 in the complex case is given below:

Proposition 3.1.10. Let $x=\left(a_{1}, a_{2}, \ldots, a_{m}\right)$ and $y=\left(b_{1}, b_{2}, \ldots, b_{m}\right)$ in $\mathbb{C}_{m}$. The following are equivalent:

1. If there is $a|\theta|=1$ such that phase $\left(a_{i}\right)=\theta$ phase $\left(b_{i}\right)$, for some $i$, then phase $\left(a_{i} a_{j}\right)=\theta^{2}$ phase $\left(b_{i} b_{j}\right), i \neq j$ and $a_{i} \neq 0 \neq b_{i}$, for any $i$.

2. $x$ and $y$ weakly have the same phase.

Proof. (1) $\Rightarrow(2)$ : Let the index sets $I, J$ and $K$ be as in proposition 3.1.3. By (1), there is a $|\theta|=1$ such that phase $\left(a_{i}\right)=\theta$ phase $\left(b_{i}\right)$ for some $i \in K$.

Now, for any $j \in K, j \neq i$,

$$
\text { phase }\left(a_{i} a_{j}\right)=\operatorname{phase}\left(a_{i}\right) \text { phase }\left(a_{j}\right)=\theta \text { phase }\left(b_{i}\right) \text { phase }\left(a_{j}\right) \text {. }
$$

But

$$
\text { phase }\left(a_{i} a_{j}\right)=\theta^{2} \text { phase }\left(b_{i} b_{j}\right)=\theta^{2} \text { phase }\left(b_{i}\right) \text { phase }\left(b_{j}\right)
$$

Thus, it follows that phase $\left(a_{j}\right)=\theta$ phase $\left(b_{j}\right)$. Since all other coordinates of either $x$ or $y$ are zero, it follows that $x, y$ weakly have the same phase.

$(2) \Rightarrow(1)$ : By definition, there is a $|\theta|=1$ such that phase $\left(a_{i}\right)=\theta$ phase $\left(b_{i}\right)$ for all $a_{i} \neq 0 \neq b_{i}$. Now, (1) follows immediately since

$$
\text { phase }\left(a_{i} a_{j}\right)=\text { phase }\left(a_{i}\right) \text { phase }\left(a_{j}\right)
$$




\subsection{Weak Phaseless Reconstruction}

The notion of phaseless reconstruction and phase retrieval has been studied previously and many times they were implemented to satisfy the same properties. Recently it was shown in [15] that these two definitions are actually equal and we can use them interchangeably. Now we want to study if it is the same case for weak phase retrieval and weak phaseless reconstruction.

In this section, we define weak phaseless reconstruction and study its characterizations. A formal definition is given below:

Definition 3.2.1. A family of vectors $\left\{\phi_{i}\right\}_{i=1}^{n}$ in $\mathbb{H}^{m}$ does weak phaseless reconstruction if for any $x=\left(a_{1}, a_{2}, \ldots, a_{m}\right)$ and $y=\left(b_{1}, b_{2}, \ldots, b_{m}\right)$ in $\mathbb{H}^{m}$, with

$$
\left|\left\langle x, \phi_{i}\right\rangle\right|=\left|\left\langle y, \phi_{i}\right\rangle\right|, \text { for all } i \in[m],
$$

there is $a|\theta|=1$ so that

$$
a_{i}=\theta b_{i}, \text { for all } i=1,2, \ldots, m, \text { for which } a_{i} \neq 0 \neq b_{i} \text {. }
$$

In particular, $\left\{\phi_{i}\right\}_{i=1}^{n}$ does phaseless reconstruction for vectors having all non-zero coordinates.

Note that if $\Phi=\left\{\phi_{i}\right\}_{i=1}^{n} \in \mathbb{R}^{m}$ does weak phaseless reconstruction, then it does weak phase retrieval. The converse is not true in general:

Let $x=\left(a_{1}, a_{2}, \ldots, a_{m}\right)$ and $y=\left(b_{1}, b_{2}, \ldots, b_{m}\right)$. If $\Phi=\left\{\phi_{i}\right\}_{i=1}^{n} \in \mathbb{R}^{m}$ does weak phase retrieval and $\left|\left\{i \mid a_{i} b_{i} \neq 0\right\}\right|=2$ then $\Phi$ may not satisfy weak phaseless reconstruction. If $a_{i} a_{j}=b_{i} b_{j}$ where $a_{i} b_{i} \neq 0$ and $a_{j} b_{j} \neq 0$ then we certainly cannot conclude in general that $\left|a_{i}\right|=\left|b_{i}\right|$ (see Example 3.3.1). 
Theorem 3.2.2. If $x=\left(a_{1}, a_{2}, \ldots, a_{m}\right)$ and $y=\left(b_{1}, b_{2}, \ldots, b_{m}\right)$ in $\mathbb{R}^{m}$. The following are equivalent:

I. There is a $\theta= \pm 1$ so that

$$
a_{i}=\theta b_{i}, \text { for all } a_{i} \neq 0 \neq b_{i} \text {. }
$$

II. We have $a_{i} a_{j}=b_{i} b_{j}$ for all $1 \leq i, j \leq m$, and $\left|a_{i}\right|=\left|b_{i}\right|$ for all $i$ such that $a_{i} \neq 0 \neq b_{i}$.

III. The following hold:

A. Either $x, y$ have weakly the same signs or they have weakly the opposite signs.

B. One of the following holds:

(i) There is $a 1 \leq i \leq m$ so that $a_{i}=0$ and $b_{j}=0$ for all $j \neq i$.

(ii) There is $a 1 \leq i \leq m$ so that $b_{i}=0$ and $a_{j}=0$ for all $j \neq i$.

(iii) If (i) and (ii) fail and $I=\left\{1 \leq i \leq m: a_{i} \neq 0 \neq b_{i}\right\}$, then the following hold:

(a) If $i \in I^{c}$ then $a_{i}=0$ or $b_{i}=0$.

(b) For all $i \in I,\left|a_{i}\right|=\left|b_{i}\right|$.

Proof. $(I) \Rightarrow(I I)$ : By $(I) a_{i}=\theta b_{i}$ for all $i$ such that both are non-zero, so

$$
a_{i} a_{j}=\left(\theta b_{i}\right)\left(\theta b_{j}\right)
$$

then $a_{i} a_{j}=\theta^{2} b_{i} b_{j}$. Since $\theta= \pm 1$ it follows that $a_{i} a_{j}=b_{i} b_{j}$ for all $i, j$ (that are non-zero). The second part is trivial. 
$(I I) \Rightarrow(I I I):$

(A) This follows from Proposition 3.1.3.

(B) (i) Assume $a_{i}=0$ but $b_{i} \neq 0$. Then for all $j \neq i$ we have $a_{i} a_{j}=0=b_{i} b_{j}$ and so $b_{j}=0$.

(ii) This is symmetric to (i).

(iii) If (i) and (ii) fail, then by definition, for any $i$, either both $a_{i}$ and $b_{i}$ are zero or they are both non-zero, which proves (A). (B) is immediate.

$(I I I) \Rightarrow(I)$ : The existence of $\theta$ is clear by part $A$. In part $B,(i)$ and $(i i)$ trivially imply $I$. Assume $(i i i)$ then for each $i$ such that $a_{i} \neq 0 \neq b_{i}$ and $\left|a_{i}\right|=\left|b_{i}\right|$ then $a_{i}= \pm b_{i}$.

Corollary 3.2.3. Let $\Phi$ be a frame for $\mathbb{R}^{m}$. The following are equivalent:

1. $\Phi$ does weak phaseless reconstruction.

2. For any $x=\left(a_{1}, a_{2}, \ldots, a_{m}\right)$ and $y=\left(b_{1}, b_{2}, \ldots, b_{m}\right)$ in $\mathbb{R}^{m}$, if

$$
\left|\left\langle x, \phi_{i}\right\rangle\right|=\left|\left\langle y, \phi_{i}\right\rangle\right| \text { for all } i \in[m] \text {, }
$$

then each of the equivalent conditions in Theorem 3.2.2 holds.

So far we realized that weak phase retrieval and weak phaseless reconstruction are equal. The question we can ask here is under what conditions these two terms are equal.

The following theorems provide conditions under which weak phase retrieval is equivalent to weak phaseless reconstruction. 
Proposition 3.2.4. Let $\Phi=\left\{\phi_{i}\right\}_{i=1}^{n}$ do weak phase retrieval on vectors $x=\left(a_{1}, a_{2}, \ldots, a_{m}\right)$ and $y=\left(b_{1}, b_{2}, \ldots, b_{m}\right)$ in $\mathbb{H}^{m}$. If $|I|=\left|\left\{i: a_{i} b_{i} \neq 0\right\}\right| \geq 3$ and $a_{i} a_{j}=b_{i} b_{j}$ for all $i, j \in I$, then $\Phi$ does weak phaseless reconstruction.

Proof. If $i, j, k$ are three members of $I$ with $a_{i} a_{j}=b_{i} b_{j}, a_{i} a_{k}=b_{i} b_{k}$ and $a_{k} a_{j}=b_{k} b_{j}$, then a short calculation gives

$$
a_{i}^{2} a_{j} a_{k}=b_{i}^{2} b_{j} b_{k}
$$

and hence $\left|a_{i}\right|=\left|b_{i}\right|$. This computation holds for each $i \in I$ and since $\Phi$ does phase retrieval, there is a $|\theta|=1$ so that phase $\left(a_{i}\right)=\theta$ phase $\left(b_{i}\right)$ for all $i \in[m]$. It follows that $a_{i}=\theta b_{i}$ for all $i \in[m]$.

It turns out that whenever a frame contains the unit vector basis, then weak phase retrieval and phaseless reconstruction are the same.

Proposition 3.2.5. Let the frame $\Phi=\left\{\phi_{i}\right\}_{i=1}^{n} \in \mathbb{R}^{m}$ do weak phase retrieval. If $\Phi$ contains the standard basis vectors, then $\Phi$ does phaseless reconstruction.

Proof. Let $x=\left(a_{1}, a_{2}, \ldots, a_{m}\right), y=\left(b_{1}, b_{2}, \ldots, b_{m}\right) \in \mathbb{R}^{m}$. By the definition of weak phase retrieval, $\Phi$ satisfies the equation 3.2. In particular, for $\phi_{i}=e_{i}$, the equation 3.2 implies that $\left|a_{i}\right|=\left|b_{i}\right|, \forall i \in[m]$. Hence the theorem.

We conclude this section by showing the surprising result that weak phaseless reconstruction is the same as phaseless reconstruction in $\mathbb{R}^{m}$. In other words, it is not really weak.

Theorem 3.2.6. Frames which do weak phaseless reconstruction in $\mathbb{R}^{m}$ do phaseless reconstruction. 
Proof. For a contradiction assume $\Phi=\left\{\phi_{i}\right\}_{i=1}^{n} \subset \mathbb{R}^{m}$ does weak phaseless reconstruction but fails the complement property. Then there exists $I \subset[n]$ such that $\operatorname{span}_{i \in I} \phi_{i} \neq \mathbb{R}^{m}$ and $\operatorname{span}_{i \in I^{c}} \phi_{i} \neq \mathbb{R}^{m}$.

Pick non-zero vectors $x, y \in \mathbb{R}^{m}$ such that $x \perp \operatorname{span}_{i \in I} \phi_{i} \neq \mathbb{R}^{m}$ and $y \perp \operatorname{span}_{i \in I^{c}} \phi_{i} \neq$ $\mathbb{R}^{m}$. Then for any $c \neq 0$ we have

$$
\left|\left\langle x+c y, \phi_{i}\right\rangle\right|=\left|\left\langle x-c y, \phi_{i}\right\rangle\right| \quad \text { for all } i \in[n]
$$

Now we consider the following cases where $x_{i}$ and $y_{i}$ denotes the $i$-th coordinate of the vectors $x$ and $y$.

- Case 1: $\left\{i: x_{i} \neq 0\right\} \bigcap\left\{i: y_{i} \neq 0\right\}=\emptyset$

Set $c=1$ and observe since $x \neq 0$ there exists some $i \in[n]$ such that $x_{i} \neq 0$ and $y_{i}=0$ and similarly there exists $j \in[n]$ such that $y_{j} \neq 0$ but $x_{j}=0$.

Then $x+y$ and $x-y$ have the same sign in the $i$-th coordinate but opposite signs in the $j$-th coordinate, this contradicts the assumption that $\Phi$ does weak phaseless reconstruction.

- Case 2: There exists $i, j \subset[n]$ such that $x_{i} y_{i} \neq 0$ and $x_{j}=0, y_{j} \neq 0$.

Without loss of generality, we may assume $x_{i} y_{i}>0$ otherwise consider $-x$ or $-y$. If $0<c \leq \frac{x_{i}}{y_{i}}$, then the $i$-th coordinate of $x+c y$ and $x-c y$ have the same sign whereas the $j$-th coordinates have opposite signs which contradicts the assumption.

By considering $y+c x$ and $y-c x$ this argument holds in the case that $y_{j}=0$ and $x_{j} \neq 0$.

- Case 3: $x_{i}=0$ if and only if $y_{i}=0$.

By choosing $c$ small enough, we have that $x_{i}+c y_{i} \neq 0$ if and only if $x_{i}-c y_{i} \neq 0$. 
By weak phase retrieval, there is a $|d|=1$ so that

$$
x_{i}+c y_{i}=d\left(x_{i}-c y_{i}\right)
$$

But this forces either $x_{i} \neq 0$ or $y_{i} \neq 0$ but not both which contradicts the assumption for case 3 .

It is shown in [4] that if $\Phi=\left\{\phi_{i}\right\}_{i=1}^{n}$ does phase retrieval or phaseless reconstruction in $\mathbb{H}^{m}$ and $T$ is an invertible operator on $\mathbb{H}^{m}$ then $\left\{T \phi_{i}\right\}_{i=1}^{n}$ does phase retrieval. It now follows that the same result holds for weak phaseless reconstruction. However, this result does not hold for weak phase retrieval.

Indeed, if $\phi_{1}=(1,1)$ and $\phi_{2}=(1,-1)$, then we have seen that this frame does weak phase retrieval in $\mathbb{R}^{2}$. But the invertible operator $T\left(\phi_{1}\right)=(1,0), T\left(\phi_{2}\right)=(0,1)$ maps this frame to a frame which fails weak phase retrieval. 


\subsection{Illustrative Examples}

In this section, we provide examples of frames that do weak phase retrieval in $\mathbb{R}^{3}$ and $\mathbb{R}^{4}$. As seen earlier, the vectors $(1,1)$ and $(1,-1)$ do weak phase retrieval in $\mathbb{R}^{2}$ but fail phase retrieval.

Our first example is a frame which does weak phase retrieval but fails weak phaseless reconstruction.

Example 3.3.1. We work with the row vectors of

$$
\Phi=\left[\begin{array}{l|ccc}
\phi_{1} & 1 & 1 & 1 \\
\phi_{2} & -1 & 1 & 1 \\
\phi_{3} & 1 & -1 & 1 \\
\phi_{4} & 1 & 1 & -1
\end{array}\right]
$$

Observe that the rows of this matrix form an equal norm tight frame $\Phi$ (and hence do norm retrieval ). Then if $x=\left(a_{1}, a_{2}, a_{3}\right)$ the following is the coefficient matrix where the row $E_{i}$ represents the coefficients obtained from the expansion $\left|\left\langle x, \phi_{i}\right\rangle\right|^{2}$

$$
1 / 2\left[\begin{array}{c|cccc}
a_{1} a_{2} & a_{1} a_{3} & a_{2} a_{3} & \sum_{i=1}^{3} a_{i}^{2} \\
E_{1} & 1 & 1 & 1 & 1 / 2 \\
E_{2} & -1 & -1 & 1 & 1 / 2 \\
E_{3} & -1 & 1 & -1 & 1 / 2 \\
E_{4} & 1 & -1 & -1 & 1 / 2
\end{array}\right]
$$

Then the following row operations give 


$$
\begin{aligned}
& 1 / 2\left[\begin{array}{c|cccc}
a_{1} a_{2} & a_{1} a_{3} & a_{2} a_{3} & \sum_{i=1}^{3} a_{i}^{2} \\
F_{1}=E_{1}-E_{2} & 1 & 1 & 0 & 0 \\
F_{2}=E_{3}-E_{4} & -1 & 1 & 0 & 0 \\
F_{3}=E_{1}-E_{3} & 1 & 0 & 1 & 0 \\
F_{4}=E_{2}-E_{4} & -1 & 0 & 1 & 0 \\
F_{4}=E_{1}-E_{4} & 0 & 1 & 1 & 0 \\
F_{5}=E_{2}-E_{3} & 0 & -1 & 1 & 0
\end{array}\right] \\
& 1 / 2\left[\begin{array}{c|cccc}
a_{1} a_{2} & a_{1} a_{3} & a_{2} a_{3} & \sum_{i=1}^{3} a_{i}^{2} \\
F_{1}-F_{2} & 1 & 0 & 0 & 0 \\
F_{3}+F_{4} & 0 & 0 & 1 & 0 \\
F_{5}-F_{6} & 0 & 1 & 0 & 0
\end{array}\right]
\end{aligned}
$$

Therefore we have demonstrated a procedure to identify $a_{i} a_{j}$ for all $1 \leq i \neq j \leq 3$. This shows that given $y=\left(b_{1}, b_{2}, b_{3}\right)$ satisfying $\left|\left\langle x, \phi_{i}\right\rangle\right|^{2}=\left|\left\langle y, \phi_{i}\right\rangle\right|^{2}$ then by the procedure outlined above we obtain

$$
a_{i} a_{j}=b_{i} b_{j}, \text { for all } 1 \leq i \neq j \leq 3
$$

By Proposition 3.1.3, these four vectors do weak sign retrieval in $\mathbb{R}^{3}$. However this family fails to do weak phaseless reconstruction. Observe the vectors $x=(1,2,0)$ and $y=(2,1,0)$ satisfy $\left|\left\langle x, \phi_{i}\right\rangle\right|=\left|\left\langle y, \phi_{i}\right\rangle\right|$ however do not have the same absolute value in each coordinate.

Our next example is a frame which does weak phaseless reconstruction but fails phaseless reconstruction. 
Example 3.3.1. We provide a set of six vectors in $\mathbb{R}^{4}$ which does weak phase retrieval in $\mathbb{R}^{4}$. In this case our vectors are the rows of the matrix:

$$
\Phi=\left[\begin{array}{l|cccc}
\phi_{1} & 1 & 1 & 1 & -1 \\
\phi_{2} & -1 & 1 & 1 & 1 \\
\phi_{3} & 1 & -1 & 1 & 1 \\
\phi_{4} & 1 & 1 & -1 & -1 \\
\phi_{5} & 1 & -1 & 1 & -1 \\
\phi_{6} & 1 & -1 & -1 & 1
\end{array}\right]
$$

Note that $\Phi$ fails to do phase retrieval as it requires seven vectors in $\mathbb{R}^{4}$ to do phase retrieval in $\mathbb{R}^{4}$. Given $x=\left(a_{1}, a_{2}, a_{3}, a_{4}\right), y=\left(b_{1}, b_{2}, b_{3}, b_{4}\right)$ we assume

$$
\left|\left\langle x, \phi_{i}\right\rangle\right|^{2}=\left|\left\langle y, \phi_{i}\right\rangle\right|^{2}, \text { for all } i=1,2,3,4,5,6 \text {. }
$$

Step 1: The following is the coefficient matrix obtained after expanding $\left|\left\langle x, \phi_{i}\right\rangle\right|^{2}$ for $i=1,2, \ldots, 6$.

$$
1 / 2\left[\begin{array}{c|ccccccc}
a_{1} a_{2} & a_{1} a_{3} & a_{1} a_{4} & a_{2} a_{3} & a_{2} a_{4} & a_{3} a_{4} & \sum_{i=1}^{4} a_{i}^{2} \\
E_{1} & 1 & 1 & -1 & 1 & -1 & -1 & \frac{1}{2} \\
E_{2} & -1 & -1 & -1 & 1 & 1 & 1 & \frac{1}{2} \\
E_{3} & -1 & 1 & 1 & -1 & -1 & 1 & \frac{1}{2} \\
E_{4} & 1 & -1 & -1 & -1 & -1 & 1 & \frac{1}{2} \\
E_{5} & -1 & 1 & -1 & -1 & 1 & -1 & \frac{1}{2} \\
E_{6} & -1 & -1 & 1 & 1 & -1 & -1 & \frac{1}{2}
\end{array}\right]
$$


Step 2: Consider the following row operations, the last column becomes all zeroes so we drop it and we get:

$$
\left[\begin{array}{l|cccccc}
F_{1}=\frac{1}{2}\left(E_{1}-E_{4}\right) & 0 & 1 & 0 & 1 & 0 & -1 \\
F_{2}=\frac{1}{2}\left(E_{2}-E_{5}\right) & 0 & -1 & 0 & 1 & 0 & 1 \\
F_{3}=\frac{1}{2}\left(E_{3}-E_{6}\right) & 0 & 1 & 0 & -1 & 0 & 1 \\
A_{1}=\frac{1}{2}\left(F_{1}+F_{2}\right) & 0 & 0 & 0 & 1 & 0 & 0 \\
A_{2}=\frac{1}{2}\left(F_{1}+F_{3}\right) & 0 & 1 & 0 & 0 & 0 & 0 \\
A_{3}=\frac{1}{2}\left(F_{2}+F_{3}\right) & 0 & 0 & 0 & 0 & 0 & 1
\end{array}\right]
$$

Step 3: Subtracting out $A_{1}, A_{2}$ and $A_{3}$ from $E_{1}, E_{2}, E_{3}$ and $E_{4}$, we get:

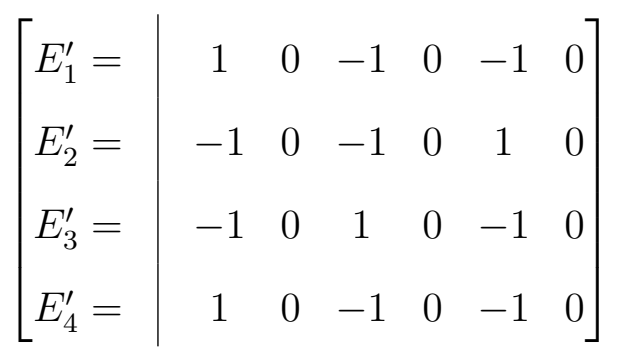

Step 4: We will show that $a_{i} a_{j}=b_{i} b_{j}$ for all $i \neq j$.

Performing the given operations we get:

$$
\left[\begin{array}{c|cccccc}
D_{1}=\frac{-1}{2}\left(E_{2}^{\prime}+E_{3}^{\prime}\right) & 1 & 0 & 0 & 0 & 0 & 0 \\
A_{2} & 0 & 1 & 0 & 0 & 0 & 0 \\
D_{2}=\frac{-1}{2}\left(E_{1}^{\prime}+E_{2}^{\prime}\right) & 0 & 0 & 1 & 0 & 0 & 0 \\
A_{1} & 0 & 0 & 0 & 1 & 0 & 0 \\
D_{3}=\frac{-1}{2}\left(E_{3}^{\prime}+E_{4}^{\prime}\right) & 0 & 0 & 0 & 0 & 1 & 0 \\
A_{3} & 0 & 0 & 0 & 0 & 0 & 1
\end{array}\right]
$$


Doing the same operations with $y=\left(b_{1}, b_{2}, b_{3}, b_{4}\right)$ we get:

$$
a_{i} a_{j}=b_{i} b_{j}, \text { for all } 1 \leq i \neq j \leq 4
$$

Remark 3.3.2. It should be noted that weak phase retrieval does not imply norm retrieval. We may use the previous example to illustrate this. Let $\Phi=\left\{\phi_{i}\right\}_{i=1}^{6}$ be as in Example 3.3.1. Suppose $\Phi$ does norm retrieval. Since there are only 6 vectors $\Phi$ fails the complement property. Now, take $x=(1,1,-1,1) \perp\left\{\phi_{1}, \phi_{2}, \phi_{3}\right\}$ and $y=(1,1,1,1) \perp\left\{\phi_{4}, \phi_{5}, \phi_{6}\right\}$. Then, we have $\left|\left\langle x+y, \phi_{i}\right\rangle\right|=\left|\left\langle x-y, \phi_{i}\right\rangle\right|$ for all $i=1,2, \ldots 6$. From the definition of norm retrieval, this implies $\|x+y\|=\|x-y\|$ which is a contradiction. 


\section{Bibliography}

[1] P.G. Casazza, R.G. Lynch, A brief introduction to Hilbert space frame theory and its applications, submitted.

[2] P. G. Casazza and G. Kutyniok,Finite Frames: Theory and Applications,Birkh auser, 2013.

[3] Cahill, Jameson, Peter G. Casazza, Jesse Peterson, and Lindsey Woodland. "Phase retrieval by projections." arXiv preprint arXiv:1305.6226 (2013).

[4] S. Bahmanpour, J. Cahill, P. G. Casazza, J. Jasper, and L.M. Woodland, Phase retrieval and norm retrieval, (2014). arXiv preprint arXiv:1409.8266.

[5] Botelho-Andrade, Sara, Peter G. Casazza, Shani Jose Dorsa Ghoreishi, and Janet C. Tremain. "Weak Phase Retrieval." Compressed Sensing and its Applications (2017): 221.

[6] Casazza, Peter G., Dorsa Ghoreishi, Shani Jose, and Janet C. Tremain. "Norm retrieval and phase retrieval by projections." Axioms 6, no. 1 (2017): 6 .

[7] R. Balan, P.G. Casazza, and D. Edidin, On Signal Reconstruction Without Phase, Appl. and Compt. Harmonic Analysis, 20, No. 3, (2006) 345-356. 
[8] A.S. Bandeira, J. Cahill, D. Mixon and A.A. Nelson, Saving phase: injectivity and stability for phase retrieval, Appl. and Comput. Harmonic Anal, 37 (1) (2014) 106-125.

[9] Edidin, D. Projections and Phase retrieval. Appl. Comput. Harmonic Anal. 2017, 42, 350-359.

[10] Cahill, J., Casazza, P.G., Jasper, J. and Woodland, L.M., 2015, September. Phase retrieval. In Wavelets and Sparsity XVI (Vol. 9597, p. 95970O). International Society for Optics and Photonics.

[11] R. H. Bates and D. Mnyama, The status of practical Fourier phase retrieval, Advances in Electronics and Electron Physics, 67 (1986), 1-64.

[12] C. Becchetti and L. P. Ricotti, Speech recognition theory and $C++$ implementation, Wiley (1999).

[13] G. Kutyniok, K. A. Okoudjou, F. Philipp, and E. K. Tuley Scalable Frames Lin. Alg. Appl. 438 (5) (2013), 2225-2238.

[14] B. Bodmann and N. Hammen, Stable Phase retrieval with low redundancy frames, Preprint. arXiv:1302.5487.

[15] S. Botelho-Andrade, P. G. Casazza, H. Van Nguyen, J. C. Tremain, Phase retrieval versus phaseless reconstruction, J. Math Anal. Appl., 436 (1), (2016) $131-137$.

[16] A. Conca, D. Edidin, M. Hering, and C. Vinzant, An algebraic characterization of injectivity of phase retrieval, Appl. Comput. Harmonic Anal, 38 (2) (2015) 346-356.

[17] J. Drenth, Principles of protein x-ray crystallography, Springer, 2010. 
[18] J. R. Fienup, Reconstruction of an object from the modulus of its fourier transform, Optics Letters, 3 (1978), 27-29.

[19] J. R. Fienup, Phase retrieval algorithms: A comparison, Applied Optics, 21 (15) (1982), 2758-2768.

[20] T. Heinosaari, L. Maszzarella, and M.M. Wolf, Quantum tomography under prior information, Comm. Math. Phys. 318 No. 2 (2013) 355-374.

[21] L. Rabiner, and B. H. Juang, Fundamentals of speech recognition, Prentice Hall Signal Processing Series (1993).

[22] M. A. Hasankhani Fard, Norm Retrievable Frames in Rn, Elect. J. Lin. Alg. 31 (1) (2016), 425-432.

[23] J. M. Renes, R. Blume-Kohout, A. J. Scott, and C. M. Caves, Symmetric Informationally Complete Quantum Measurements, J. Math. Phys., 45 (2004), 2171-2180.

[24] C. Vinzant, A small frame and a certificate of its injectivity, Preprint. arXiv: $1502.0465 \mathrm{v} 1$.

[25] C. Vinzant, A small frame and a certificate of its injectivity, in 2015 International Conference on Sampling Theory and Applications (SampTA), IEEE, 2015, pp. 197-200.

[26] I. Peng and S. Waldron, Signed frames and Hadamard products of Gram matrices, Linear Algebra Appl. 347 (2002), 131-157.

[27] A. Rahimi and P. Balazs, Multipliers for p-Bessel sequences in Banach spaces, Integral Equations Oper. Theory 68 (2) (2010), 193-205. 


\section{VITA}

Dorsa Ghoreishi was born in Tehran, Iran. She attended Khaje Nasir Toosi University of Technology in Tehran for her baccalaureate degree in applied mathematics. In the same year she started her PhD program at the University of Missouri. She also attained her masters degree during her $\mathrm{PhD}$ program with master thesis on weak phase retrieval. Throughout her time at the University of Missouri, she has worked with Professor Peter Casazza at the Frame Research Center. 